\title{
TRANSLATION SURFACES WITH NO CONVEX PRESENTATION
}

\author{
SAMUEL LELIÈVRE AND BARAK WEISS
}

\begin{abstract}
We give infinite lists of translations surfaces with no convex presentations. We classify the surfaces in the stratum $\mathcal{H}(2)$ which do not have convex presentations, as well as those with no strictly convex presentations. We show that in $\mathcal{H}(1,1)$, all surfaces in the eigenform loci $\mathcal{E}_{4}, \mathcal{E}_{9}$ or $\mathcal{E}_{16}$ have no strictly convex presentation, and that the list of surfaces with no convex presentations in $\mathcal{H}(1,1) \backslash\left(\mathcal{E}_{4} \cup \mathcal{E}_{9} \cup \mathcal{E}_{16}\right)$ is finite and consists of square-tiled surfaces. We prove the existence of non-lattice surfaces without strictly convex presentations in all of the strata $\mathcal{H}^{(\mathrm{hyp})}(g-1, g-1)$.
\end{abstract}

A translation surface is a union of polygons with pairs of parallel edges identified by translation, up to cut and paste equivalence. These structures have been intensively studied in recent years in connection with questions in group theory, geometry, complex analysis, and dynamics - we recall the definitions in $\$ 1$ and refer to the recent surveys $\mathrm{MT}, \mathrm{ZO}$, for detailed definitions and discussions of work on translation surfaces. The cut and paste equivalence means that the same translation surface has many presentations as unions of polygons. It is well-known (see Vi, §12]) that a connected translation surface may be presented as a single polygon with an even number of edges identified pairwise. A natural question is whether there is such a presentation, in which the polygon can be taken to be convex. In his 1992 Hayashibara Forum Lecture, Veech V2] was the first to exhibit surfaces with no presentations as convex polygons. We review his examples and give new ones in $\$ 2$. These include the Veech double $n$-gons, the Escher staircases, the Ward examples, and the Bouw-Möller examples.

Translation surfaces are naturally grouped in strata, which are moduli spaces of translation surfaces for which the combinatorics of singularities is fixed. Although the constructions in $\$ 2$ yield infinitely many examples, they only give rise to finitely many in each stratum. In this paper we take up the question of classifying surfaces with no convex presentations in the simplest strata, namely the genus two strata $\mathcal{H}(2)$ and $\mathcal{H}(1,1)$. It will be useful to distinguish between convex and strictly convex polygons. Although, strictly speaking, polygons are never strictly convex, we will say that a polygon is strictly convex if none of its vertices is in the convex hull of the other vertices; equivalently, the internal angles at vertices of the polygon are strictly less than $\pi$. In making this definition we always assume that polygons have no spurious vertices, in other words, edges of polygons are not strictly contained in larger straight segments on which the identification map of edges extends continuously. We will see that strictly convex presentations only arise in the hyperelliptic components of the strata $\mathcal{H}(2 g-2)$ and $\mathcal{H}(g-1, g-1)$.

Let $G \stackrel{\text { def }}{=} \mathrm{SL}_{2}(\mathbb{R})$. There is a natural $G$-action on each stratum of translation surfaces, and, as noted by Veech [V2], the property of having no (strictly) convex 
presentations is $G$-invariant. Moreover the property of having no strictly convex presentations is closed. Thus the question of classifying surfaces without convex presentations is intimately connected to the question of understanding the closures of $G$-orbits. Our results crucially rely on the work of McMullen [Mc2] describing the possible orbit closures for the $G$-action on $\mathcal{H}(2)$ and $\mathcal{H}(1,1)$.

We remind the reader that by work of Calta $[\mathrm{C}$ ] and McMullen [Mc1, Mc3] (see $\$ 3$ for a review), the $G$-orbits of lattice surfaces in $\mathcal{H}(2)$ are in bijective correspondence with symbols $D, D_{0}, D_{1}$, where $D \geq 5$ is the discriminant, and is congruent to a square $\bmod 4$, and where for $9<D \equiv 1 \bmod 8$ there are symbols $D_{0}, D_{1}$ corresponding to even and odd spin structures. Some surfaces in $\mathcal{H}(1,1)$ have Jacobians which are eigenforms for an action of an order of discriminant $D$ in the field $\mathbb{Q}(\sqrt{D})$. We will denote the locus of such surfaces in $\mathcal{H}(1,1)$ by $\mathcal{E}_{D}$. In this paper, by an arithmetic surface we mean a translation cover of a torus branched over one point, i.e. a surface which is in the $G$-orbit of a square-tiled surface. Note that lattice surfaces which are eigenforms of discriminant $D$, where $D$ is a square, are arithmetic. The following are our main results.

Theorem 1. Up to the G-action, the list of surfaces in $\mathcal{H}(2)$ which have no strictly convex presentation are those corresponding to symbols

$$
5,9,12,16,17_{1}, 21,25_{0}, 25_{1}, 32,36,41_{0}, 45,49_{1}, 64,77,81_{1} .
$$

$U p$ to the $G$-action, the list of surfaces in $\mathcal{H}(2)$ with no convex presentation are those corresponding to symbols

$$
5,12,17_{1}, 21,32,41_{0}, 45,77 .
$$

Theorem 2. Let $\mathcal{E}_{4,9,16} \stackrel{\text { def }}{=} \mathcal{E}_{4} \cup \mathcal{E}_{9} \cup \mathcal{E}_{16} \subset \mathcal{H}(1,1)$. Then:

(i) There is no surface in $\mathcal{E}_{4,9,16}$ with a strictly convex presentation.

(ii) If $D \notin\{4,9,16\}(D \equiv 0,1 \bmod 4)$, then the eigenform locus $\mathcal{E}_{D}$ contains a surface with a strictly convex presentation.

(iii) Any surface in $\mathcal{H}(1,1)$ which has no strictly convex presentation is either arithmetic or belongs to $\mathcal{E}_{4,9,16}$.

(iv) The number of $G$-orbits of arithmetic surfaces with no strictly convex presentations in $\mathcal{H}(1,1) \backslash \mathcal{E}_{4,9,16}$ is finite.

(v) For each $D \in\{4,9,16\}$, there are surfaces in $\mathcal{E}_{D}$ which are not lattice surfaces and have no convex presentations.

The space $\mathcal{E}_{4,9,16}$ parametrizes torus covers of low degree, and assertion (i) is a special case of a more general statement (see Proposition 42 which exhibits loci of torus covers with no strictly convex presentation for any genus $g \geq 2$. Assertion (v) resolves a question of Veech [V2, Question 5.2]. Using Theorem 2, in \$10 we answer a more refined question of Veech. Our proof of (iii) is ineffective, and it would be interesting to write down the full list of examples of the arithmetic surfaces as in (iii). We exhibit two such examples, in discriminant 49, in 2.1 .

We briefly describe the proofs of our results. Clearly a convex polygon contains a strip running through it which connects parallel edges on opposite sides of the polygon. When $g \geq 2$, this shows that a translation surface with a convex presentation has a simple cylinder, i.e. a cylinder which is bounded on both sides by a single saddle connection. The existence of a simple cylinder is a nontrivial restriction and this observation underlies our construction (in $\$ 2$ of many new examples 
of surfaces with no convex presentations. In genus two, the dynamical results of McMullen allow us to conclude that a surface with no convex presentation is either a lattice surface or an eigenform. By further results of Calta and McMullen, the core direction of the simple cylinder is a completely periodic direction. By work of McMullen, Calta and Bainbridge, completely periodic directions on genus two surfaces are described in terms of prototypes, which are explicit presentations of completely periodic surfaces. There are finitely many prototypes for each discriminant $D$ in $\mathcal{H}(2)$, and in $\mathcal{H}(1,1)$, prototypes also depend on two continuous real parameters. In $\$ 4$ we show that there is a canonical polygonal presentation associated with each prototype, which may or may not be convex. We call this the canonical polygon associated with the prototype. A strictly convex presentation, if it exists, must be the canonical polygon of some prototype.

To prove Theorem [1, we first exhibit explicitly, for all $D \geq 200$, a particular prototype whose canonical polygon is convex. For the remaining cases $5 \leq D \leq 200$ we use a computer program, discussed in \$5.4 to list all prototypes and test their canonical polygon for convexity. Figure 1 shows two prototypes in $D=12$ and $D=13$ and shows the corresponding canonical polygon. Using this program we obtain the list (1). Sample output of our computer program is shown in Figure 11. Appealing to results of Hubert, Lelièvre and McMullen we find that lattice surfaces which are arithmetic do admit a one-cylinder convex presentation. We use this to obtain the list 2 .

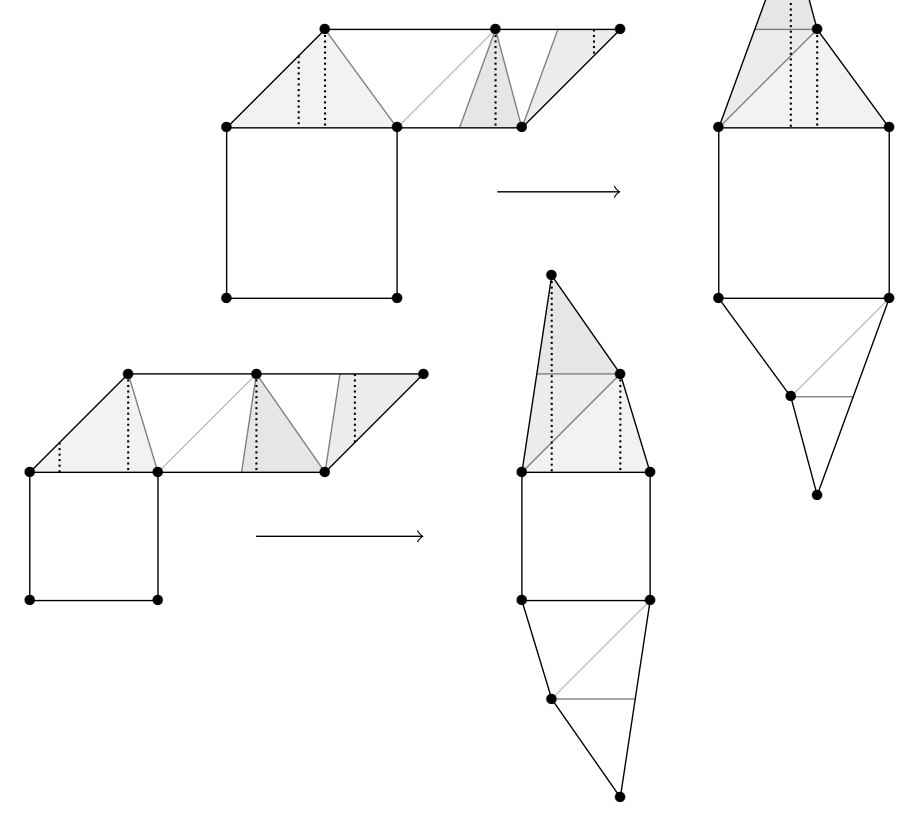

Figure 1. Canonical polygon of prototype $(D, a, b, c, e)$ : Prototype $(12,1,3,1,0)$ yields a non-convex polygon.

Prototype $(13,1,2,1,-1)$ yields a strictly convex polygon. 
To prove Theorem $2(\mathrm{i})$ we use the fact that surfaces in $\mathcal{E}_{4,9,16}$ admit a branched covering map of a twice punctured torus, of low degree, to rule out strictly convex canonical polygons. Note that in $\mathcal{H}(1,1)$ the number of prototypes for a fixed $D$ is no longer finite so we cannot use a brute force computer search as in the case $\mathcal{H}(2), D \leq 200$. For Theorem 2(ii), we discuss separately the discriminants $D$ which do or do not appear in the list (1). If $D$ does not appear in (1), the existence of a strictly convex polygon in $\mathcal{H}(2)$ forces the existence of a nearby strictly convex polygon of discriminant $D$ in $\mathcal{H}(1,1)$. For the remaining cases of $D \neq 4,9,16$ which do appear in (1) we give explicit strictly convex canonical polygons for some prototypes (these were found by a computer search). Assertion (iii) of Theorem 2 follows from assertion (ii) and McMullen's dynamical results for the $G$-action on $\mathcal{H}(1,1)$.

The proof of the finiteness statement in Theorem 2(iv) relies on a deep recent finiteness result of EM, EMM]. To prove Theorem 2(v) we show that convex but not strictly convex presentations may be 'easily destroyed' by a local perturbation in $\mathcal{E}_{D}$. Since there are no strictly convex presentations in $\mathcal{E}_{4,9,16}$, this implies that the set of surfaces in $\mathcal{E}_{4,9,16}$ with no convex presentations at all must be of full measure, with respect to the natural measure on these loci.

Acknowledgments: Our interest in the question of convex presentations of translation surfaces was inspired by Thierry Monteil's 'banana peel argument' Mo. We thank Matt Bainbridge for useful discussions. We thank Pat Hooper for permission to use his picture (Figure 4) of the Bouw-Möller surfaces, and for useful discussions about these surfaces. This research was supported by the ANR projet blanc GEODYM, Israel Science Foundation grant 190/08 and European Research Council grant DLGAPS 279893.

\section{General Remarks}

A cylinder for a translation surface is a topological annulus which is isometric to $(0, h) \times \mathbb{R} / c \mathbb{Z}$, and is not properly contained in a larger cylinder. Here $h$ and $c$ are respectively the height and circumference of the cylinder. The image of $\left\{x_{0}\right\} \times \mathbb{R} / c \mathbb{Z}$ under such an isometry is called a waist curve. A cylinder is always bounded by saddle connections parallel to the waist curve. A cylinder is called simple if both of its boundary consists of a single saddle connection.

Proposition 3. Suppose $M$ is a translation surface of genus at least two, which has a convex presentation as a $2 n$-gon. Then $M$ contains a simple cylinder $C$, and there is a direction $\theta$ transverse to the circumference of $C$, such that $\theta$ is the direction of a saddle connection contained in $C$ passing between its boundary edges (i.e. a 'diagonal'), and any path in $M$ in direction $\theta$ either lies in a saddle connection or intersects both boundary components of $C$.

Furthermore, if the $2 n$-gon is strictly convex, then there is a hyperelliptic involution of $M$ fixing $C$, all paths in $M$ in direction $\theta$ intersect any boundary component of $E$ of $C$, and the return map $E \rightarrow E$ obtained by moving in direction $\theta$ is an interval exchange on $n-1$ intervals, corresponding to the 'inversive permutation' $i \mapsto n-i$.

Proof. Denote the convex $2 n$-gon representing $M$ by $P$. Since $M$ has genus at least two, $n \geq 4$. Let $e_{1}, \ldots, e_{2 n}$ denote the edges of $P$, in cyclic counterclockwise order. Let $\theta_{i}$ denote the angle that the edge $e_{i}$ makes with the horizontal direction where 
the $e_{i}$ are oriented so that $P$ is on their left. We consider $\theta_{i}$ modulo $2 \pi$ and write $\theta^{\prime}<\theta$ if the short path along the boundary of the circle of directions from $\theta^{\prime}$ to $\theta$ is in the counterclockwise direction. Then convexity (strict convexity) is equivalent to $\theta_{i} \leq \theta_{i+1}$ (resp. $\theta_{i}<\theta_{i+1}$ ) for all $i$. For each $i$ we denote by $k=k(i)$ the index for which $e_{i}$ and $e_{k}$ are identified in $M$.

The convex hull of $e_{j}$ and $e_{k}, k=k(j)$ is a parallelogram in the plane. Denote its sides $e_{j}, u_{j}, e_{k}, v_{j}$ in the usual order. This parallelogram maps to a cylinder $C$ in $M$, which is simple if and only if the sides $u_{j}$ and $v_{j}$ contain no vertices of $P$ in their interior. It is clear from convexity that any path in $M$ in direction $\theta=\theta_{j}$ intersects the boundary edges of $C$, so to prove the first assertion we have only to find $j$ for which $C$ is simple.

Suppose $C$ is not simple and suppose with no loss of generality that $u_{j}$ contains vertices in its interior, then $k-j \geq 3$ and

$$
\theta_{j}<\theta_{j+1}=\cdots=\theta_{k-1}<\theta_{k} .
$$

Assume first that $k-j \geq 4$, that is an edge of $P$ has three consecutive parallel edges. Then replacing $j$ with $j+2$, we see from (3) that neither $u_{j+2}$ nor $v_{j+2}$ can contain vertices in their interior, i.e. the parallelogram $\operatorname{conv}\left(e_{j+2}, e_{k(j+2)}\right)$ maps to a simple cylinder in $M$, as required. Now assume $k=j+3$. Then the equations

$$
0 \leq \theta_{i+1}-\theta_{i}<\pi, \theta_{j+1}=\theta_{k(j+1)}, \theta_{j}=\pi+\theta_{j+3}, \theta_{i}=\pi+\theta_{k(i)}
$$

imply that the angles $\theta_{i}$ assume only the four values $\left\{\theta_{j}, \theta_{j+1}, \pi+\theta_{j}, \pi+\theta_{j+1}\right\}$, i.e. $P$ is a parallelogram. If it has three consecutive parallel edges we are done as in the previous case. Otherwise $n=4$ and in each direction there are two consecutive parallel edges. Since there are no spurious vertices on $P$, in this case the gluing must be $k(i)=i+4 \bmod 8$, and one sees that for any $j, \operatorname{conv}\left(e_{j}, e_{k(j)}\right)$ maps to a simple cylinder, as required.

Now suppose $P$ is strictly convex. We claim that

$$
\forall j, \quad k(j)=j+n \bmod 2 n .
$$

Indeed, if this is not true then (replacing $j$ with $k(j)$ and re-indexing cyclically) we would have for some $r<n$ :

$$
\theta_{1}<\cdots<\theta_{r}=\pi+\theta_{1}<\cdots<\theta_{2 n}<\theta_{1} .
$$

On the other hand, since $r<n$ there is some $j_{0}$ so that both $j_{0}$ and $k\left(j_{0}\right)$ are in $\{r+1, \ldots, 2 n\}$. This implies $\theta_{j_{0}}=\pi+\theta_{k\left(j_{0}\right)}$, contradicting [5. The remaining assertions of the Proposition follow easily from (4), where the involution is the symmetry with respect to the center of the parallelogram representing the cylinder C.

The following is an immediate consequence:

Corollary 4. Suppose that $M$ is a translation surface with a presentation as a strictly convex $2 n$-gon, with $n \geq 4$. Then:

- All vertices of the $2 n$-gon are singularities for the translation structure.

- If $n$ is even then $M$ belongs to the hyperelliptic connected component of the stratum $\mathcal{H}(2 g-2)$ where $g=\frac{n}{2}$ is the genus of $M$.

- If $n$ is odd then $M$ belongs to the hyperelliptic connected component of the stratum $\mathcal{H}(g-1, g-1)$ where $g=\frac{n-1}{2}$ is the genus of $M$. 
Remark 5. It was proved by Kontsevich and Zorich $[\mathrm{KZ}$ that in every connected component of every stratum there is a dense set of surfaces with a one-cylinder presentation. In particular having a convex presentation places no constraint on the topology of the surface. Corollary 4 shows that having a strictly convex presentation does entail a topological restriction.

We will need another consequence of Proposition 3.

Corollary 6. Suppose $M$ is a translation surface of genus $g \geq 2$ represented by a strictly convex polygon $P$ with opposite sides identified, and suppose $C$ is a simple cylinder in $M$ joining opposite sides of $P$. Let $\delta$ be the saddle connection joining the top and bottom of $C$ which is represented by two opposite segments on the boundary of $P$. Then any saddle connection parallel to $\delta$ is longer than $\delta$, and $M$ has no other simple cylinder which is parallel to $C$ and contains a diagonal parallel to $\delta$.

In particular, a surface with a strictly convex presentation does not have a nontrivial translation automorphism.

Proof. Assume with no loss of generality that $C$ is represented by a central square in $P$ with opposing vertical edges identified. Since $g \geq 2, P$ does not cover the entire surface $M$, and since $P$ is strictly convex, any vertical saddle connection in $P$ must pass through $C$ at least once and have endpoints disjoint from the top and bottom edges of $C$; in particular its length is greater than the height of $C$. Thus, if $C^{\prime}$ is another cylinder in $M$ which is parallel to $C$, with a diagonal $\delta^{\prime}$ parallel to $\delta$, the interior of $\delta^{\prime}$ must intersect $C$, that is the interiors of $C$ and $C^{\prime}$ intersect. This is not possible for parallel cylinders.

This proves the first assertion, and the second assertion follows by considering the image of $C$ under a translation automorphism.

The following simple observation, noted by Veech [V2, is at the heart of our discussion. Its proof is immediate from the definitions and is left as an exercise to the reader.

Proposition 7. Let $\mathcal{H}$ be a connected component of a stratum of translation surface. Then the subsets $\mathcal{H}_{\mathrm{nc}}, \mathcal{H}_{\mathrm{nsc}}$ of $\mathcal{H}$ consisting respectively of surfaces with no convex (resp., no strictly convex) presentations are $G$-invariant, and $\mathcal{H}_{\mathrm{nsc}}$ is closed.

\section{A ZOO OF EXAMPles}

In this section we give four infinite lists of examples of surfaces which do not admit convex presentations. These will all be lattice surfaces, i.e. surfaces $M$ for which the $G$-orbit $G M$ is closed. Equivalently, their Veech group $\Gamma=\{g \in G$ : $g M=M\}$ is a lattice in $G$. As Veech showed in [V1, on a lattice surface, the direction of a core curve of a cylinder is always completely periodic, all non-critical leaves in that direction are closed. Moreover the $\Gamma$-orbits of completely periodic directions are in bijective correspondence with the cusps of the quotient $G / \Gamma$, i.e. with conjugacy classes of maximal parabolic subgroups of $\Gamma$. In proving that a lattice surface has no convex presentation, our strategy will be to examine the various completely periodic directions on $M$, and show that they cannot be the direction of a core curve of a simple cylinder as in Proposition 3. 
We start with the Veech double $n$-gon, obtained by taking $n \geq 5$ and forming two regular $n$-gons $\Delta_{1}, \Delta_{2}$, where $\Delta_{2}$ is the reflection of $\Delta_{1}$ in (any) one of its side, and gluing each edge of $\Delta_{1}$ with the parallel edge of $\Delta_{2}$. See Figure 2 .
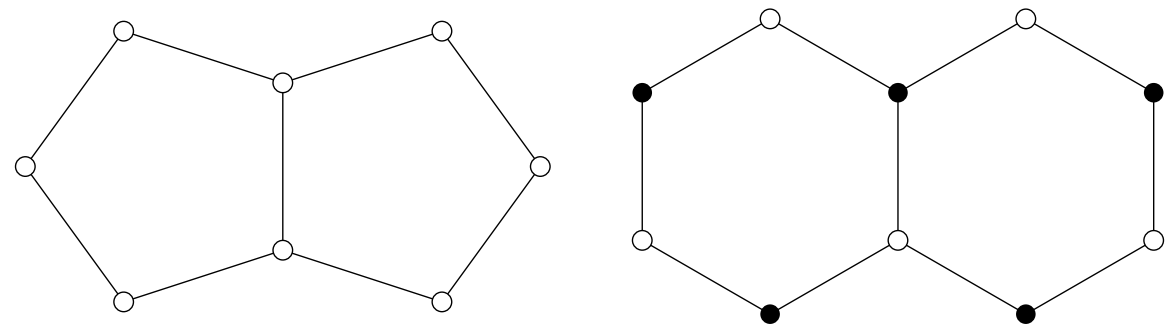

FiguRe 2. In a double $(2 g+1)$-gon, the horizontal and vertical directions are in the unique class of parabolic directions, while in a double $(2 g+2)$-gon, they represent the two classes of parabolic directions.

Proposition 8. The Veech double n-gons have no convex presentations.

Remark 9. Veech [V2] proved this assertion for $n$ odd and asserted its validity for all n, without proof. Our argument is different from that of Veech.

Proof. Veech [V1] showed that the double $n$-gon surfaces are lattice surfaces, and computed their Veech group, showing that it has either one cusp (when $n$ is odd) or two (when $n$ is even). If $n$ is odd, in the cylinder decomposition corresponding to the unique cusp, the only simple cylinder lies at the left and right sides of the double $n$-gon (vertical direction in Figure 2) and this cylinder does not intersect all leaves in the direction of the corresponding diagonal. Thus the necessary condition of Proposition 3 is not satisfied. If $n$ is even then one cylinder decomposition has no simple cylinders at all, and the other has one simple cylinder, treated as in the previous case of $n$ odd.

Now let $n \geq 3$. The Escher staircase is obtained by a cyclic gluing of squares as in Figure 3 . That is, if the squares are labelled $1, \ldots, 2 n$, then square $2 k+1$ is attached to square $2 k+2$ along its horizontal sides, and to square $2 k$ along its two vertical sides (all calculations modulo $2 n$ ).

Proposition 10. The Escher staircases have no convex presentations.

Proof. One shows using Schmithüsen's algorithm $\underline{S}$ ] that there are two cusps, corresponding to the horizontal and unit slope directions in Figure 3. These directions have no simple cylinders.

Ward $[\mathrm{W}$ ] constructed translation surfaces by applying the 'unfolding procedure' to the billiards in the $\left(\frac{\pi}{2 n}, \frac{\pi}{n}, \frac{(2 n-3) \pi}{2 n}\right)$ triangles, and proved that the corresponding surfaces are lattice surfaces. See Figure 3

Proposition 11. The Ward examples have no convex presentations. 

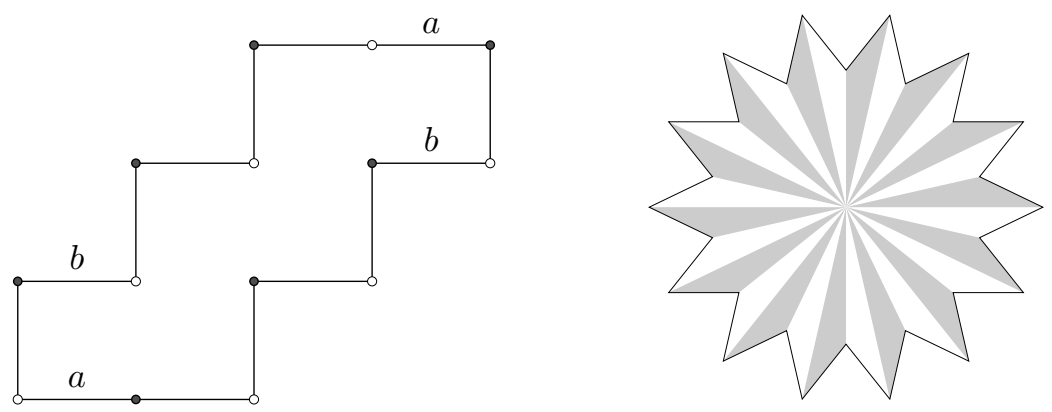

Figure 3. Left: Escher stairs with $g$ steps, odd $g$ (here $g=3$ ). Right: Unfolding of Ward's $\left(\frac{\pi}{14}, \frac{\pi}{7}, \frac{11 \pi}{14}\right)$ triangle.

Proof. As proved by Ward, in his examples the Veech group has one cusp. Therefore the horizontal direction in Figure 3 represents the unique completely periodic direction (up to the $\Gamma$-action). To locate the cylinders in this direction one simply draws all horizontal segments starting at vertices of the polygon in the figure; this analysis reveals that there are no simple cylinders in the horizontal direction. Therefore the Ward surfaces have no simple cylinders at all.

Bouw and Möller $[\mathrm{BM}]$ found an infinite sequence of lattice surfaces $\mathrm{BM}_{m, n}$.

Proposition 12. When $n \geq m \geq 4$ and $n, m$ are not both even, the surface $\mathrm{BM}_{m, n}$ has no convex presentation.

Proof. Hooper $[\mathrm{H}]$ described the Bouw-Möller examples in terms of a decomposition into semi-regular polygons; see also $[\mathrm{Wr}$, where it is shown that Hooper's surfaces in fact coincide with $\mathrm{BM}_{m, n}$. The work of $[\mathrm{BM}, \mathrm{H}, \mathrm{Wr}$ ] also showed that the Veech group of $\mathrm{BM}_{m, n}$ is either the $(m, n, \infty)$ triangle group (when $n>m$ ), or the $(2, n, \infty)$-triangle group (when $n=m$ are odd), and hence these examples have a unique (up to the Veech group action) cylinder decomposition pattern. Hence any simple cylinder, if it existed, would have to be horizontal in a direction of a semi-regular polygon decomposition. It can be checked from Hooper's description (see Figure 4) that when $n \geq m \geq 4$ and $m, n$ are not both even, the surfaces $\mathrm{BM}_{m, n}$ have no simple cylinders.

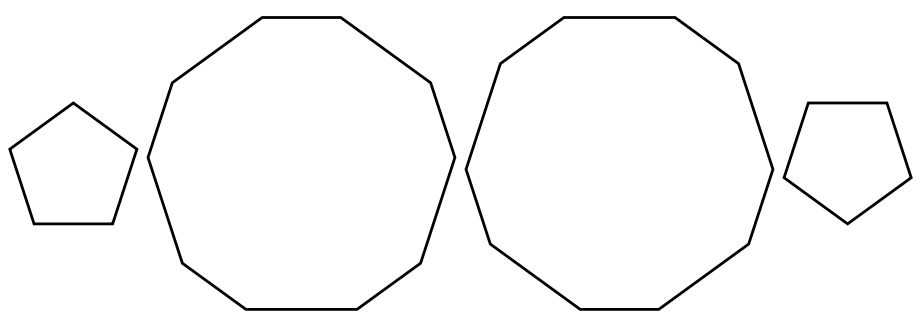

Figure 4. Hooper's presentation of the Bouw-Möller surface $\mathrm{BM}_{4,5}$. Each edge is identified with an edge in a neighboring polygon. 
Remark 13. The property of having no simple cylinder is also G-invariant and closed. It would be interesting to classify all surfaces without simple cylinders. A similar closed and $G$-invariant property is having no semisimple cylinder, i.e. a cylinder which is bounded on at least one side by a single saddle connection.

2.1. Some square-tiled examples. We conclude this zoological survey with four interesting square-tiled surfaces. No menagerie is complete without the legendary eierlegende Wollmilchsau and ornithorhynque (see $\mathrm{Ma}$ ). One of the many surprising features of these notoriously eccentric square tiled surfaces, is the lack of simple cylinders. In particular they have no convex presentations.

Proposition 14. The surface made of four squares (see the left hand side of Figure 5) has no convex presentation. Any square-tiled surface in $\mathcal{H}(1,1)$ made of seven squares or fewer (see the two surfaces on the right hand side of Figure 5) has no strictly convex presentations.
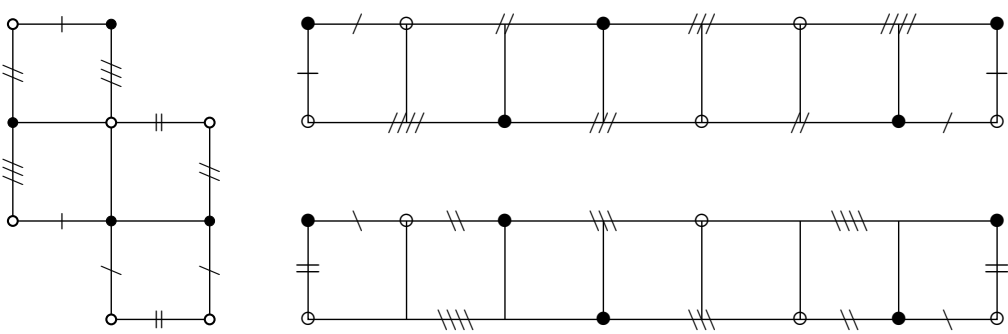

FiguRE 5. Left: this 4-square-tiled surface in $\mathcal{E}_{16}$ has no onecylinder direction and no convex presentation.

Right: representatives for the two $\operatorname{SL}(2, \mathbb{R})$-orbits of 7 -square-tiled surfaces in $\mathcal{E}_{49}$. Both have no strictly convex presentation.

Proof. For the first assertion, checking the $\mathrm{SL}_{2}(\mathbb{Z})$-orbit (see $[\mathbf{S}]$ ) one finds that the $G$-orbit of this surface has two cusps, none of whose corresponding cylinder decompositions is a one-cylinder decomposition. Only one of the cusps has a simple cylinder $C$, and one can verify that the foliation in the direction of any diagonal of $C$ has leaves which do not cross $C$ and are not on saddle connections. So by Proposition 3, the surface has no convex presentation.

For the second assertion, let $M \in \mathcal{H}(1,1)$ be square-tiled, and suppose $C$ is a simple cylinder of a convex presentation, as in Proposition 3. We claim that $C$ has area at least four (where we normalize area so that each square is of unit area). Indeed, replacing $M$ by its image under an element of $\mathrm{SL}_{2}(\mathbb{Z})$ we can represent $C$ as a rectangle in the plane with corners at integer points, where all vertices of $M$ remain at integer points, such that the two boundary edges of $C$ are horizontal. In this situation, a generalized diagonal is an edge crossing $C$ from top to bottom, where it is allowed to wrap around the circumference of $C$. For rectangles of area less than four, straight lines starting at integer points in the plane, in the directions of generalized diagonals, can intersect the boundary of $C$ in at most three points. However according to Proposition 3 , there must be four intersection points, corresponding to the discontinuity points of the interval exchange. This proves the claim. 
Now let $P$ be a convex polygon representing $M$. Since $M \in \mathcal{H}(1,1), P$ is a decagon, and $P \backslash C$ is a union of two quadrilaterals. Identifying their edges which are boundary components of $C$, we obtain a new strictly convex polygon $P^{\prime}$. The vertices of $P^{\prime}$ are integer points and $P^{\prime}$ is an octagon of area at most three. Recall Pick's formula $\left[\mathrm{P}\right.$, which says that the area of $P^{\prime}$ is $i+b / 2-1$, where $i$ is the number of interior integer points and $b$ is the number of integer points along the edges. By construction $b \geq 8$, so in fact $b=8, i=0$ and the area of $P^{\prime}$ is exactly three. Label the sides of $P^{\prime}$ by vectors $v_{1}, \ldots, v_{8}$, cyclically in the counterclockwise direction. Applying $\mathrm{SL}_{2}(\mathbb{Z})$ again we can assume that $v_{1}, v_{2}$ meet at the origin and lie respectively along the $y$-axis and $x$-axis. Since the eight vertices are the only integer points on sides, $v_{1}, v_{2}$ are of unit length. By strict convexity, the internal angle between $v_{8}$ and $v_{1}$ is more than $\pi / 2$ and so is the internal angle between $v_{2}$ and $v_{3}$. This forces $(1,1)$ to be an interior point of $P^{\prime}$, a contradiction.

\section{A SUMMARY OF RESULTS ON GENUS TWO SURFACES}

In this section we summarize results about genus two surfaces which will be crucial for our discussion. These results incorporate work of many authors, notably McMullen. We refer the reader to the surveys $[\mathrm{HS}, \mathrm{ZO}$ ] for a more detailed discussion.

All surfaces considered in this section will be of genus two. Given a positive integer $D$ let $\mathcal{O}_{D}$ be the order of discriminant $D$ in the field $\mathbb{Q}(\sqrt{D})$. We say that $M$ is an eigenform surface of discriminant $D$ if $\mathcal{O}_{D}$ embeds in the endomorphism ring of its Jacobian.

Theorem 15 (McMullen). Any G-orbit in $\mathcal{H}(2)$ is either closed or dense.

Theorem 16 (Veech, Calta, McMullen). The following are equivalent for $M \in$ $\mathcal{H}(2)$ :

- $M$ is a lattice surface.

- $M$ is an eigenform surface.

- Any saddle connection direction is completely periodic.

- Any cylinder direction is completely periodic.

Suppose the above hold and let $D$ be the corresponding discriminant. Then:

- $M$ is arithmetic if and only if $D$ is a square.

- The number of $G$-orbits of lattice surfaces of discriminant $D \geq 5$ is:

0 if $D \equiv 2,3 \bmod 4$.

1 if $D \equiv 0,4,5 \bmod 8$.

2 if $D \equiv 1 \bmod 8$.

We will refer to the $G$-orbits of lattice surfaces in $\mathcal{H}(2)$ by their discriminant $D$; in case $D \equiv 1 \bmod 8$ we will write $D_{0}, D_{1}$ to distinguish the two cases in (6) (these are distinguished by the parity of their spin structure, see [Mc3]). We will abuse notation by referring to the symbols $D_{0}, D_{1}$ as discriminants.

Theorem 17 (Calta-McMullen prototypes). Suppose $\theta$ is a completely periodic direction on a lattice surface in $\mathcal{H}(2)$ of discriminant $D$, such that the corresponding cylinder decomposition has two cylinders. Then one of the cylinders $C$ is simple, and there are integers $a, b, c, e$ such that:

$$
\operatorname{gcd}(a, b, c, e)=1, D=e^{2}+4 b c, b>0, c>0, c+e<b, 0 \leq a<b .
$$




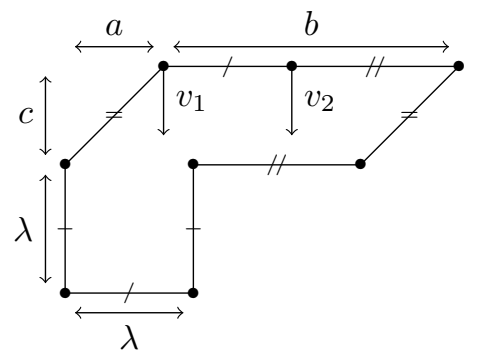

Figure 6. Dimensions and labels for a prototype in $\mathcal{H}(2)$.

After applying an affine map which makes the core curve of $C$ horizontal, a saddle connection crossing $C$ vertical, and the height of $C$ equal to its circumference, and equal to $\lambda=\frac{e+\sqrt{D}}{2}$, the surface is presented as in Figure 6 .

Furthermore, for each $D$ there are only finitely many choices of a, b, c, e satisfying (7), and for each such choice, the surface of Figure 6 is a surface of discriminant $D$ with a completely periodic horizontal direction. In case $D \equiv 1 \bmod 8$, the parity of its spin structure is

$$
\frac{e-f}{2}+(c+1)(a+b+a b) \bmod 2,
$$

where $f$ depends on $D$.
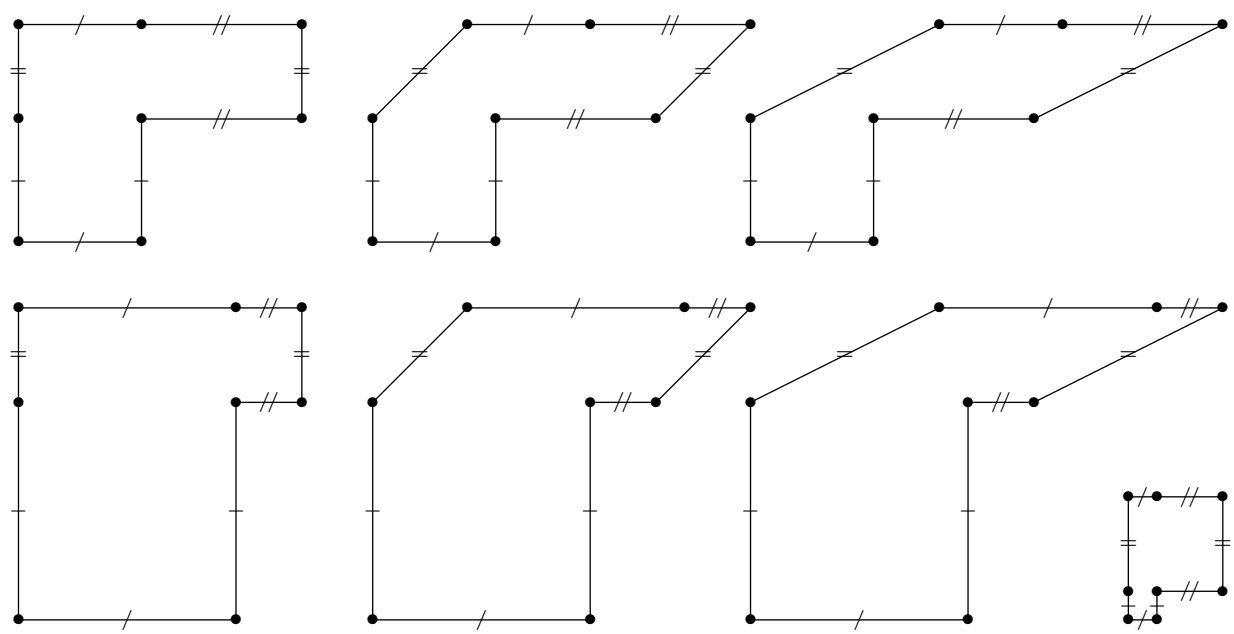

Figure 7 . The seven prototypes for discriminant 13 in $\mathcal{H}(2)$.

Remark 18. Our notation differs slightly from that of McMullen [Mc3], who took $a<\operatorname{gcd}(b, c)$ and introduced an additional parameter $t$. Our a corresponds to $a+t c$ in McMullen's notation. McMullen's notation was better suited to his purpose of listing the different prototypes which correspond to the same cusp of the corresponding G-orbit-information which will not be relevant to our discussion. 
Let $\mathcal{E}_{D}$ be the eigenform locus of discriminant $D$, i.e. the subset of $\mathcal{H}(1,1)$ consisting of eigenform surfaces of discriminant $D$. We say that two surfaces $M_{1}, M_{2} \in \mathcal{H}(1,1)$ are rel equivalent if there is an identification of their homology groups $H_{1}\left(M_{i} ; \mathbb{Z}\right)$ such that corresponding 1-cycles have the same holonomy. This equivalence relation is generated by a local surgery which moves one singularity with respect to the other without changing absolute periods. See $\$ 8$ for more details.

Theorem 19 (Calta, McMullen). The eigenform locus $\mathcal{E}_{D}$ is closed and invariant under both the $G$-action and rel-equivalence. The following are equivalent for $M \in$ $\mathcal{H}(1,1)$ :

- $M$ is an eigenform surface.

- Any cylinder direction is completely periodic.

Theorem 20 (McMullen). For $D \geq 4, D \equiv 0,1 \bmod 4$, each $\mathcal{E}_{D}$ is a connected 5-dimensional suborbifold of $\mathcal{H}(1,1)$ which is equipped with a unique smooth $G$ invariant ergodic measure. This measure is finite.

Theorem 21 (McMullen). For any surface in $M \in \mathcal{H}(1,1)$, one of the following (mutually exclusive) assertions holds:

- $M$ is an eigenform surface of discriminant $D$ and is a lattice surface. In this case $M$ is either arithmetic, and $D$ is a square, or $M$ is in the orbit of the regular decagon, and $D=5$.

- $M$ is an eigenform surface of discriminant $D$ and its $G$-orbit is dense in $\mathcal{E}_{D}$.

- $M$ is not an eigenform surface and its $G$-orbit is dense in $\mathcal{H}(1,1)$.

Given $x \in \mathbb{R}$ and $b>0$ we write

$$
R_{b}(x) \stackrel{\text { def }}{=} x-\left\lfloor\frac{x}{b}\right\rfloor \cdot b,
$$

that is $R_{b}(x)$ is the unique element in $x+b \mathbb{Z} \cap[0, b)$. The following result is proved in $\mathrm{Mc} 3, \mathrm{~B}$

Theorem 22 (McMullen, Bainbridge). Suppose $\theta$ is a completely periodic direction on an eigenform surface of discriminant $D$ in $\mathcal{H}(1,1)$, such that the corresponding cylinder decomposition has three cylinders, or two cylinders with one simple cylinders. Then there are integers $a, b, c, e$, and real numbers $x, y$ such that $a, b, c, e$ satisfy (7), and

$$
0 \leq x<b-\lambda, \quad 0 \leq y<\frac{\lambda c}{\lambda+c}
$$

After applying an affine map as in Theorem 17, and setting

$$
s \stackrel{\text { def }}{=} R_{b}\left(a+x\left(1+\frac{c}{\lambda}\right)\right), t \stackrel{\text { def }}{=} c-y\left(1+\frac{c}{\lambda}\right)
$$

the surface is presented as in Figure 8. The number of cylinders in the cylinder decomposition is two if and only if $y=0$.

Furthermore, suppose $a, b, c, e, x, y$ satisfy (7) and (10), and define $s, t$ via (11). Then the surface of Figure $[6$ is a surface of discriminant $D$ with a completely periodic horizontal direction. 


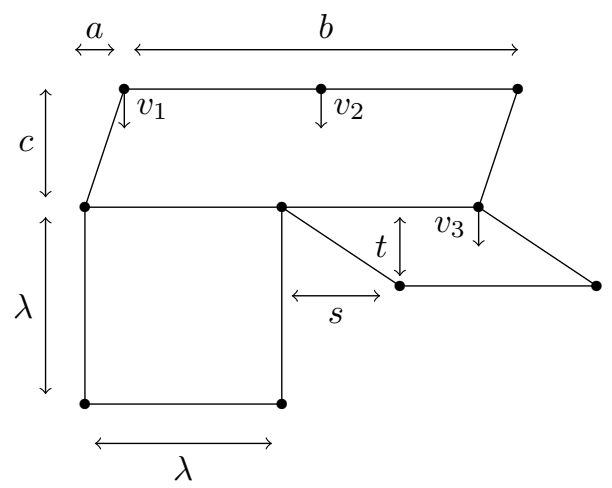

Figure 8. Dimensions and labels for a prototype in $\mathcal{H}(1,1)$.

Note that when $y>0$ there are two simple cylinders in the presentation, the taller one is represented by a $\lambda \times \lambda$ square. By an abuse of notation which should cause no confusion, we will refer to the latter simple cylinder as the simple cylinder of the prototype.

3.1. One-cylinder presentations. We say that $M$ has a one-cylinder presentation if it can be presented as one cylinder with gluings along its boundary edges. The following useful result is well-known to those who know it well. For an elegant proof of Hubert, see [HLM, Lemma 5.3]. For a generalization, see [CS].

Proposition 23. If $M$ is a lattice surface with a one-cylinder presentation then $M$ is arithmetic.

We will also need the following statement, which follows from $\mathrm{Mc}$, $\mathrm{HL}$ :

Proposition 24 (Hubert-Lelièvre, McMullen). Any arithmetic surface in $\mathcal{H}(2)$ has a one-cylinder presentation.

Remark 25. The conclusion of Proposition 24 fails in $\mathcal{H}(1,1)$. For an example, see the surface portrayed on the left hand side of Figure 5 .

3.2. Finiteness result of Eskin-Mirzakhani-Mohammadi. In recent breakthrough work [EM, EMM], severe restrictions were found on the possible $G$-invariant measures and $G$-orbit-closures in any stratum $\mathcal{H}$. We will need the following:

Theorem 26 (see [EMM], Theorem 2.2). For any stratum $\mathcal{H}$, any closed $G$ invariant subset is a union of finitely many orbit-closures.

As an immediate consequence of Proposition 7 and Theorems 15, 16, 21 and 26. we have:

Corollary 27. In $\mathcal{H}(2)$, the set of surfaces with no strictly convex presentations consists of finitely many $G$-orbits of lattice surfaces. In $\mathcal{H}(1,1)$, the set of surfaces with no strictly convex presentations consists of finitely many G-orbits of lattice surfaces and finitely many eigenform loci. 
Remark 28. One could derive Corollary 27 without appealing to [EMM], using McMullen's results [Mc2] and an analysis of horocycle trajectories close to the supports of the $G$-invariant measures of $\mathcal{H}(1,1)$. We omit the details.

\section{The CANONiCAL POLYGON}

In this section we will start with a surface of genus two presented via the prototypes of $\$ 3$, and give an alternative presentation of the same surface via what we will call the canonical polygon. This will be an octagon (resp. a decagon) with edge identifications giving rise to a surface in $\mathcal{H}(2)$ (resp. $\mathcal{H}(1,1)$ ). The construction of the canonical polygon is explicit and algorithmic. We will show that if a surface of genus two admits a strictly convex presentation, then it is a canonical polygon for some prototype. We will discuss the two strata $\mathcal{H}(2)$ and $\mathcal{H}(1,1)$ separately.

4.1. Canonical polygons in $\mathcal{H}(2)$. Let $M$ be a lattice surface in $\mathcal{H}(2)$ presented via a prototype as in Theorem 17, and let $C$ be the simple cylinder of the prototype. Recall that $C$ is normalized so that its circumference and height are both equal to $\lambda$, its circumference is horizontal, and there is a vertical saddle connection passing through $C$ from bottom to top. We will say that the prototype is nondegenerate for $M$ if every non-critical vertical leaf passes through $C$ infinitely many times, and every critical leaf passes through the interior of $C$ at least once. Otherwise we will say the prototype is degenerate for $M$. Let $\sigma$ be the horizontal saddle connection which is the top boundary segment of $C$.

Proposition 29. The prototype with parameters $a, b, c, e, \lambda, D$ is nondegenerate for $M$ if and only if there are non-negative integers $k$ and $\ell$ such that the following hold:

and

$$
R_{b}(k a) \in(0, \lambda) \text { and } R_{b}(j a) \notin[0, \lambda] \text { for } j=1, \ldots, k-1,
$$

In this case the return map to $\sigma$ going upward along vertical leaves is an interval exchange on three intervals, and the corresponding permutation is the 'inversive permutation' $i \mapsto 4-i$.

Proof. Nondegeneracy is equivalent to all minimal components of the vertical flow intersecting the interior of $C$. Equivalently, all vertical separatrices going down from the singularity pass through $C$. Let $k$ and $\ell$ denote respectively the number of times the downward vertical separatrices marked $v_{1}, v_{2}$ in Figure 6 , pass through the nonsimple cylinder in the prototype, before reaching the interior of $C$. The horizontal coordinate of a point changes by an addition of $a$ whenever the separatrix passes through the non-simple cylinder without reaching $C$. This leads to the formulae (12) and (13).

The two points where the two prongs touch $\sigma$ are the two discontinuities of the interval exchange which is the return map to $\sigma$. Thus the interval exchange has two discontinuities, i.e. three intervals of continuity. The center of $C$ is a Weierstrass point. Symmetry of the vertical foliation with respect to the hyperelliptic involution implies that the permutation is inversive, as claimed.

The canonical polygon for the prototype is constructed as follows (see Figure 9): For each of the three intervals of continuity of the interval exchange in Proposition 29 construct the strip going up along vertical leaves from the top of $C$ to the 
bottom of $C$, and number them from left to right. The singularity of $M$ appears on the right-hand boundary edge of the first strip, on both boundary edges of the second strip, and on the left-hand boundary edge of the third strip. Cut the strips by segments joining the singularity to itself, one in each strip, and rearrange. The canonical polygon is the resulting octagon.

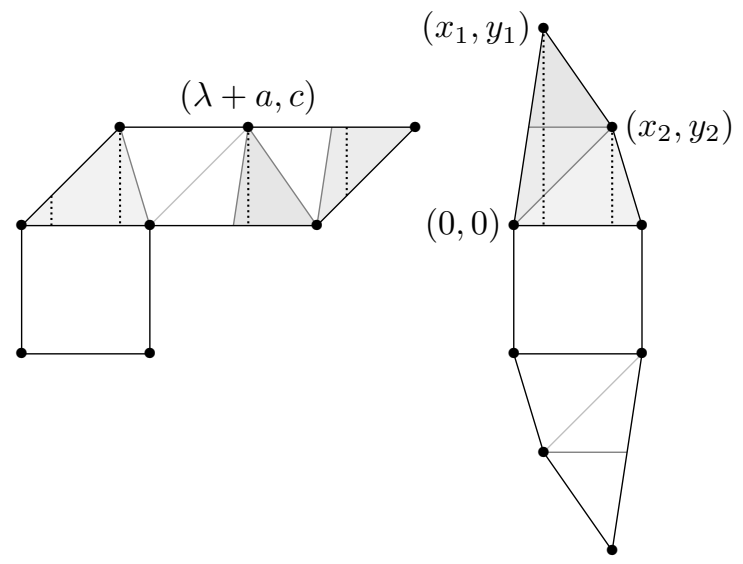

Figure 9. Flowdown lines and canonical polygon of a prototype: $(D, a, b, c, e)=(13,1,2,-1) ; \lambda=\frac{\sqrt{13}-1}{2}$.

Let $\left(x_{1}, y_{1}\right),\left(x_{2}, y_{2}\right)$ denote respectively the holonomies of the segment going from the top left-hand corner of $C$ to the singularity of $M$, by following the top of $C$ until the first (respectively second) discontinuity of the interval exchange transformation described in Proposition 29, and then up to the singularity along the right hand edge of the first (resp. second) strip.

Proposition 30. Let $k$ and $\ell$ be as in $(12)$ and 13 . Then

$$
\left(\begin{array}{l}
x_{1} \\
y_{1}
\end{array}\right)=\left(\begin{array}{c}
R_{b}(\lambda+\ell a) \\
\ell c
\end{array}\right), \quad\left(\begin{array}{c}
x_{2} \\
y_{2}
\end{array}\right)=\left(\begin{array}{c}
R_{b}(k a) \\
k c
\end{array}\right),
$$

and $x_{1}<x_{2}$. The canonical polygon is strictly convex if and only if

$$
x_{2} y_{1}-x_{1} y_{2}>0, x_{2} y_{1}-x_{1} y_{2}>\lambda\left(y_{1}-y_{2}\right) .
$$

Proof. The points $\left(x_{i}, y_{i}\right), i=1,2$ are vertices of the octagon in light of Proposition 29 To find out which of these two points is to the left of the other, we check how these vertices are identified with the vertices in the prototype diagram; we see that in the octagon, the vertical prong going down from the vertex at $\left(x_{2}, y_{2}\right)$ is reached from the vertical prong at $(0,0)$ by moving counterclockwise for an angle of $2 \pi$. The same description is valid for the vertical prong at $(\lambda+a, c)$, thus they represent the same prong.

The two inequalities in (15) correspond to internal angles less than $\pi$ at each of the two vertices $\left(x_{1}, y_{1}\right),\left(x_{2}, y_{2}\right)$. The other six vertices follow automatically from these via the hyperelliptic involution, and from the inequalities $0<x_{1}<x_{2}<$ $\lambda$.

Our interest in the canonical polygon is motivated by the following: 
Proposition 31. If $M$ is a lattice surface in $\mathcal{H}(2)$ and has a strictly convex presentation, then there is a prototype which is nondegenerate for $M$, for which the given convex polygon presentation coincides with the canonical polygon.

Proof. Let $\mathcal{P}$ be the convex polygon representing $M$ and let $C$ be a simple cylinder as in Proposition 3 . Then the direction of the circumference of $C$ is completely periodic by Theorem 16. Consider the prototype corresponding to this direction via Theorem 17. The convexity of $\mathcal{P}$ implies that the prototype is nondegenerate for $M$. The definition of $\left(x_{i}, y_{i}\right)$ given in the discussion preceding Proposition 30 now shows that $\mathcal{P}$ coincides with the canonical polygon for this prototype.

4.2. Canonical polygons in $\mathcal{H}(1,1)$. We proceed analogously to 4.1. making the required adjustments. Let $M$ be an eigenform surface in $\mathcal{H}(2)$. Suppose $M$ is presented via a prototype as in Theorem 22, and let $C$ be the simple cylinder of the prototype. As before, we will say that the prototype is nondegenerate for $M$ if every non-critical vertical leaf passes through $C$ infinitely many times, and every critical leaf passes through the interior of $C$ at least once, and degenerate otherwise. Note that the vertical direction need no longer be a completely periodic direction for $M$. Let $\sigma$ be the horizontal saddle connection which is the top boundary segment of $C$. Let $R_{b}$ be as in (9), define $s, t$ via (11), and define

$$
R:[\lambda, b] \rightarrow[0, b), \quad R(z) \stackrel{\text { def }}{=} R_{b}(z-x+s) .
$$

Proposition 32. The prototype with parameters $a, b, c, e, \lambda, D, x, y$ is nondegenerate for $M$ if and only if $x \neq 0, s \notin\{0, \lambda, b-\lambda\}$ and there are non-negative integers $k, \ell, m$ such that the following hold:

$$
\begin{gathered}
R^{k}(s) \in(0, \lambda) \text { and } R^{j}(s) \notin[0, \lambda] \text { for } j=1, \ldots, k-1, \\
R^{\ell}(s+\lambda) \in(0, \lambda) \text { and } R^{j}(s+\lambda) \notin[0, \lambda] \text { for } j=1, \ldots, \ell-1
\end{gathered}
$$

and

$$
R^{m}(b) \in(0, \lambda) \text { and } R^{j}(b) \notin[0, \lambda] \text { for } j=1, \ldots, m-1 .
$$

In this case the return map to $\sigma$ going upward along vertical leaves is an interval exchange on four intervals, and the corresponding permutation is the 'inversive permutation' $i \mapsto 5-i$.

Proof. The map $R$ in $(16)$ is the return map to the bottom of the non-simple cylinder of the prototype, for those $z$ which do not begin along the boundary with the simple cylinder $C$. As before, nondegeneracy is equivalent to the requirement that all vertical separatrices going down from the singularity pass through the interior of $C$. Let $k, \ell, m$ denote respectively the number of times the downward vertical separatrices $v_{1}, v_{2}, v_{3}$ respectively in Figure 10 , pass through the non-simple cylinder in the prototype, before reaching the interior of $C$. This leads to the formulae (17), 18) and (19).

The three points in the interior of $\sigma$ are the three discontinuities of the interval exchange which is the return map to $\sigma$. Thus the interval exchange has four intervals of continuity. Counting Weierstrass points one sees that there must be a Weierstrass point in the center of $C$. In particular $C$ is fixed by the involution. Symmetry of the vertical foliation with respect to the hyperelliptic involution implies that the permutation is inversive, as claimed. 

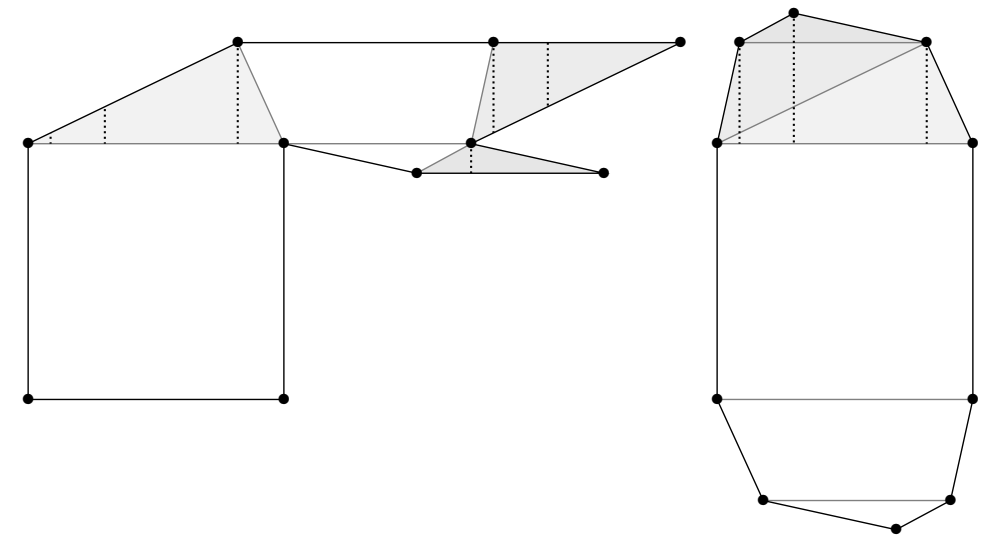

FIgURE 10. From prototype to canonical decagon in $\mathcal{H}(1,1)$.

The canonical polygon for the prototype is constructed as before. It is a decagon. Let $\left(x_{1}, y_{1}\right),\left(x_{2}, y_{2}\right),\left(x_{3}, y_{3}\right)$ denote respectively the holonomies of the segment going from the top left-hand corner of $C$ to the singularity of $M$, by following the top of $C$ until the first (respectively second and third) discontinuity of the interval exchange transformation described in Proposition 29, and then up to the singularity along the right hand edge of the first (resp. second and third) strip.

The following propositions are proved analgously to Propositions 30 and 31 . We leave the proofs to the reader.

Proposition 33. Let $k$ and $\ell$ be as in (12) and (13). Then

$$
\left(\begin{array}{l}
x_{1} \\
y_{1}
\end{array}\right)=\left(\begin{array}{c}
R^{\ell}(s+\lambda) \\
\ell(y+t)+t
\end{array}\right), \quad\left(\begin{array}{l}
x_{2} \\
y_{2}
\end{array}\right)=\left(\begin{array}{c}
R^{m}(b) \\
m(y+t)
\end{array}\right), \quad\left(\begin{array}{l}
x_{3} \\
y_{3}
\end{array}\right)=\left(\begin{array}{c}
R^{k}(s) \\
k(y+t)+t
\end{array}\right)
$$

and $x_{1}<x_{2}<x_{3}$. The canonical polygon is strictly convex if and only if

$$
\begin{aligned}
& x_{2} y_{1}-x_{1} y_{2}>0, \\
& x_{2} y_{3}-x_{3} y_{2}+x_{3} y_{1}-x_{1} y_{3}+x_{1} y_{2}-x_{2} y_{1}>0, \\
& x_{2} y_{3}-x_{3} y_{2}+\lambda\left(y_{2}-y_{3}\right)>0 .
\end{aligned}
$$

Proposition 34. If $M$ is a lattice surface in $\mathcal{H}(1,1)$ and has a strictly convex presentation, then there is a prototype which is nondegenerate for $M$, for which the given convex polygon presentation coincides with the canonical polygon.

\section{Proof of Theorem 1}

The main result of this section is:

Proposition 35. The list (1) is the complete list of discriminants for which there is no prototype for which the canonical polygon is strictly convex.

Deduction of Theorem 1 from Proposition 35 . The stratum $\mathcal{H}(2)$ contains a surface with a strictly convex presentation, viz. the regular octagon. Therefore there is an 
open subset of $\mathcal{U}$ of $\mathcal{H}(2)$ consisting of surfaces with a strictly convex presentation. By Theorem 15. if $M$ is not a lattice surface then its $G$-orbit intersects $\mathcal{U}$ and therefore $M$ has a strictly convex presentation. Therefore the first assertion of Theorem 1 follows from Propositions 31 and 35. For the second assertion, note that (2) is obtained from (1) by removing all $D$ which are square. Thus it suffices to show that if $D$ is a square, $M$ has a convex presentation, and if $D$ is not a square and the surface of discriminant $D$ has a convex presentation, it must be strictly convex. The first part of this claim follows from Proposition 24. Now suppose $M$ has a convex presentation which is not strictly convex, then the corresponding polygon $\mathcal{P}$ must be either a parallelogram or a hexagon with some vertices in the interiors of edges. If $\mathcal{P}$ is a parallelogram then the surface has a one-cylinder presentation, and $D$ is a square by Proposition 24. If $\mathcal{P}$ is a hexagon, let $\theta$ be the direction of a side of $\mathcal{P}$. The flow in direction $\theta$ is completely periodic by Theorem 16 and the return map to a diagonal of the hexagon is an interval exchange on two intervals. This means that this return map is a rational rotation, i.e. $\mathcal{P}$ maps to a one-cylinder presentation with waist curve in direction $\theta$. Again using Proposition 24 we find that $D$ is a square.

5.1. Computations for even $D$. Given even $D$ let $\kappa$ be such that

$$
(2 \kappa)^{2}<D \leq(2 \kappa+2)^{2} \text {. }
$$

Set $e \stackrel{\text { def }}{=} 2 \kappa-6$ and

$$
b \stackrel{\text { def }}{=} \frac{D-e^{2}}{4}
$$

so that

$$
6 \kappa-9<b \leq 8 \kappa-8
$$

Now define

$$
\lambda \stackrel{\text { def }}{=} \frac{e+\sqrt{D}}{2}
$$

so that

$$
2 \kappa-3<\lambda \leq 2 \kappa-2 \text {. }
$$

Let $a$ be the unique integer in the interval $\left[\frac{b+\lambda}{3}-1, \frac{b+\lambda}{3}\right)$.

Proposition 36. The following hold when $\kappa \geq 7$ :

(i) $\frac{b+\lambda}{3}-\frac{b-\lambda}{2}>1$.

(ii) $3 \lambda<b$.

(iii) $b-\lambda<2 a<b$.

(iv) $3 a>b$.

(v) $a>\lambda$.

(vi) $\lambda+a<b$.

(vii) $3 a-b<\lambda$.

Proof. It follows from 24 and 26 that

$$
\frac{b+\lambda}{3}-\frac{b-\lambda}{2}>\frac{2 \kappa-7}{6}
$$


which implies (i), since $\kappa \geq 7$. To prove (ii), define $x \stackrel{\text { def }}{=} \sqrt{D}$. We need to show $\frac{3(\sqrt{D}+e)}{2}<\frac{D-e^{2}}{4}$. Plugging in $e=2 \kappa-6$ and $x=\sqrt{D}$, this is equivalent to

$$
x^{2}-6 x-4 \kappa^{2}+12 \kappa>0 \text { for } x \in(2 \kappa, 2 \kappa+2],
$$

which is easily proved using the quadratic formula. The right hand side of (iii) follows from (ii) and the definition of $a$. The left hand side follows from the definition of $a$ and (i). Since $\lambda \geq 3$, (iv) follows from the definition of $a$. The inequality (v) follows from (ii) and (iv), and inequality (vi) follows from (iii) and (v). Inequality (vii) is immediate from the definition of $a$.

5.2. Computations for odd $D$. Given odd $D$ let $\kappa$ be such that

$$
(2 \kappa-1)^{2}<D \leq(2 \kappa+1)^{2}
$$

Set $e_{1} \stackrel{\text { def }}{=} 2 \kappa-7, e_{2} \stackrel{\text { def }}{=} 2 \kappa-9$ and define $b_{1}, b_{2}$ via $(23)$, using $e_{1}, e_{2}$ respectively, so that

$$
6 \kappa-12<b_{1} \leq 8 \kappa-12, \quad 8 \kappa-20<b_{2} \leq 10 \kappa-20 .
$$

Define $\lambda_{1}, \lambda_{2}$ via 25 so that

$$
2 \kappa-5<\lambda_{2} \leq 2 \kappa-4<\lambda_{1} \leq 2 \kappa-3 .
$$

Let $a_{1}$ be the unique integer in the interval $\left[\frac{b+\lambda}{3}-1, \frac{b+\lambda}{3}\right)$ and let $a_{2}$ be the unique integer in the interval $\left[\frac{b_{2}+\lambda_{2}}{4}-1, \frac{b_{2}+\lambda_{2}}{4}\right)$.

Proposition 37. Inequalities (i) - vii) from Proposition 36 hold for $a_{1}, b_{1}, \lambda_{1}$ when $\kappa \geq 7$. If $\kappa \geq 5$ then:

(i) $\frac{b_{2}+\lambda_{2}}{4}-\frac{b_{2}-\lambda_{2}}{3}>1$.

(ii) $4 \lambda_{2}<b_{2}$.

(iii) $b_{2}-\lambda_{2}<3 a_{2}<b_{2}$.

(iv) $4 a_{2}>b_{2}$

(v) $a_{2}>\lambda_{2}$.

(vi) $\lambda_{2}+2 a_{2}<b_{2}$.

(vii) $4 a_{2}-b_{2}<\lambda_{2}$.

Proof. Very similar to the proof of Proposition 36, and we leave the details to the reader.

\subsection{There are strictly convex presentations for all sufficiently large $D$.}

Corollary 38. For any $D \geq 200$, the lattice surface of discriminant $D$ has a strictly convex presentation.

Proof. Suppose first that $D$ is even. Let $a, b, e$ be as in Proposition 36 , and set $c \stackrel{\text { def }}{=} 1$. Then one easily checks that (7) holds so that $D, a, b, c, e$ is a prototype. Inequalities (v), (iii), (iv) and (vii) of Proposition 36 show that $k=3$ satisfies (12), and inequalities (iii) and (vi) imply that $\ell=2$ satisfies 13 . Finally, (15) follows from (ii).

If $D$ is odd we can use either of the prototypes $D, a_{1}, b_{1}, 1, e_{1}$ or $D, a_{2}, b_{2}, 1, e_{2}$, where in the first case we use $k=3, \ell=2$ and in the second we use $k=4, \ell=3$. It is easy to check using Proposition 37 that the hypotheses of Proposition 30 are satisfied. If $D \equiv 5 \bmod 8$ then this concludes the proof. If $D \equiv 1 \bmod 8$ there are two possible spins, so it remains to check that the two cases $D, a_{1}, b_{1}, 1, e_{1}$ 
and $D, a_{2}, b_{2}, 1, e_{2}$ correspond to different spins, and this is immediate from $e_{1}=$ $e_{2}+2, c=1$ and $(8)$.

5.4. A computer algorithm. In order to deal with the remaining cases $5 \leq D<$ 200, we have implemented the following algorithm:

(1) Given $D$, enumerate all solutions $a, b, c, e$ to (7).

(2) For each prototype $a, b, c, e$ from step 1 , do:

(a) Let $k$ be the smallest $j$ so that $R_{b}(j a) \in[0, \lambda]$, and let $\ell$ be the smallest $j$ so that $R_{b}(\lambda+a j) \in[0, \lambda]$ (cf. $(12)$ and $(13)$ ).

(b) If $\left\{R_{b}(k a), R_{b}(\lambda+\ell a)\right\} \cap\{0, \lambda\} \neq \varnothing$ then the prototype is degenerate for $M$.

(c) Check whether 15 holds. If yes, stop: the canonical octagon for this prototype is a strictly convex presentation.

(3) If 15 fails for all prototypes, then there is no strictly convex presentation.

We have implemented this algorithm on Sage. The code and output of our computation are available along with this paper at $[\mathrm{LW}$. A sample of the output of our program is below in Figure 11. Using the computation we find:

Proposition 39. For $5 \leq D<200$, there is a prototype with discriminant $D$ whose canonical octagon is convex, if and only if $D$ does not appear on the list (1).

Proof of Proposition 35. Immediate from Corollary 38 and Proposition 39.

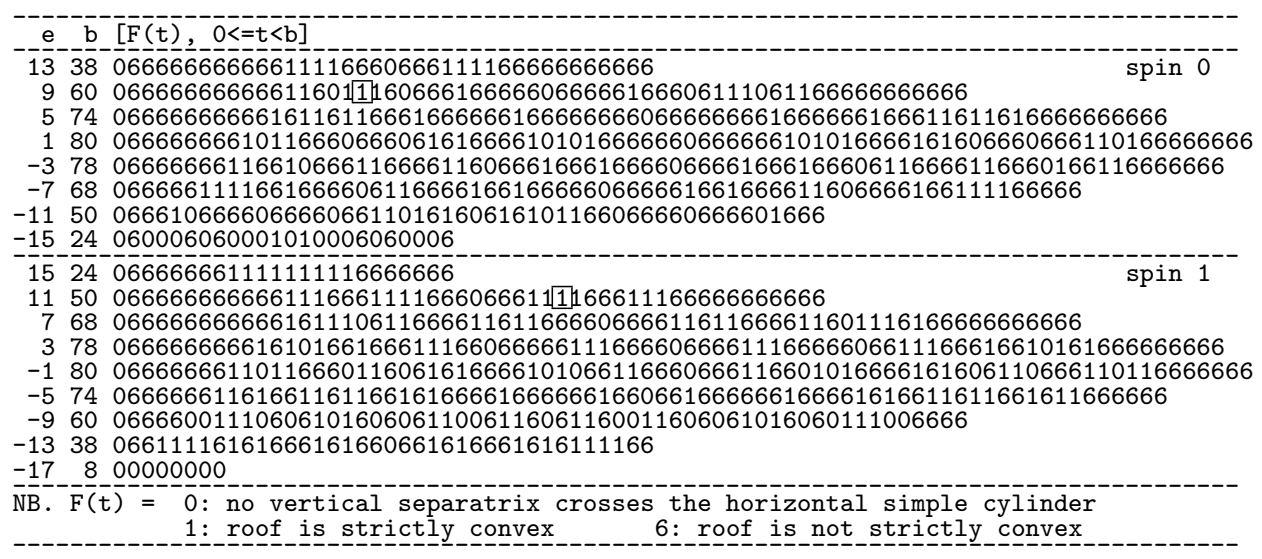

Figure 11. Flowdown algorithm output for $D=321$, both spins. The boxed 1's are the convex presentations of Corollary 38

6. The Discriminants $D=4,9,16$ in $\mathcal{H}(1,1)$

Proposition 40. No surface in $\mathcal{E}_{4,9,16}$ has a strictly convex presentation. 
Proof. Surfaces in $\mathcal{E}_{D}$ with $D=d^{2}$ admit a degree $d$ cover onto a torus with two branch points, corresponding to the distinct singularities of the surface. Suppose $M \in \mathcal{E}_{4,9,16}$ has a convex presentation and, applying the $G$-action and dilation, suppose it covers the standard square once-punctured torus with holonomy in $\mathbb{Z}^{2}$, via a degree $d$ covering map. Let $P$ be the convex decagon representing $M$, label its vertices cyclically counterclockwise, and let $P_{0}$ be the pentagon which is the convex hull of vertices labeled $1,3,5,7,9$. Then the vertices of $P_{0}$ all project to the same point on the torus. This implies that $P_{0}$ is a strictly convex pentagon in the plane, with vertices in $\mathbb{Z}^{2}$ and area strictly less than $d$. A further restriction on $P_{0}$ is that the sum of the internal angles at adjacent vertices of $P_{0}$ (i.e. vertices whose labeling on $P$ differs by 2 ) is strictly greater than $\pi$. This follows from an argument as in the last paragraph of the proof of Proposition 3. We call this the adjacent angles restriction.

Thus to conclude the proof it suffices to show that for $d=3,4,5$, there is no $P_{0}$ with the stated properties. Note that all properties are invariant under the action of $2 \times 2$ integer matrices of deteminant \pm 1 . Suppose first that there are 2 adjacent edges of $P_{0}$ which do not contain lattice points on their interior. By applying an integer matrix we can assume that three of the vertices of $P_{0}$ are $v_{1}=(0,1), v_{2}=(0,0), v_{3}=(1,0)$. We write the two additional vertices $v_{4}, v_{5}$ as $(m, n)$ and $(k, \ell)$ respectively. Then applying the adjacent angles restrictions on the various edges obtain the following inequalities:

$$
m>1, \quad n>0, \quad k>0, \quad \ell>1, \quad \ell>n, m>k, \quad n k>(m-1)(\ell-1) .
$$

From these we derive

$$
k<m<1+\frac{n k}{\ell-1} \leq 1+k,
$$

which is impossible.

For the remaining case we will use Pick's formula again (see the proof of Proposition 14. If no two adjacent edges of $P_{0}$ are without interior lattice points, then the number of lattice points is at least 8 ( 5 at the vertices and at least 3 on the edges). Additionally, we can apply an integer matrix to assuming that three of the adjacent vertices of $P_{0}$ are $(k, 0),(0,0),(0, \ell)$ with $k \geq 2$ and $\ell \geq 1$. Then the adjacent angle restrictions imply that the points $(1,1)$ and $(1,2)$ are interior points of $P_{0}$. Thus by Pick's formula the area of $P_{0}$ is at least 5 .

Remark 41. The case $D=4$ could also be proved by using Corollary 6 and noting that all surfaces in $\mathcal{E}_{4}$ have nontrivial translation automorphisms.

It is possible to extend Proposition 40 to higher genus, as follows. For $g \geq 2$, and $d \geq g$, let $\mathcal{C}^{(d)}(g-1, g-1)$ denote the Hurwitz space of surfaces in the hyperelliptic component of the stratum $\mathcal{H}(g-1, g-1)$ which admit a degree $d$ cover of a torus branched over two points. Proposition 40 corresponds to the case $g=2, d_{0}=4$ of the following statement.

Proposition 42. For any $g \geq 2$ there is $d_{0}=d_{0}(g) \geq g$ such that for $d=g, \ldots, d_{0}$, $\mathcal{C}^{(d)}(g-1, g-1)$ contains no surface with a strictly convex presentation.

Proof. We first note that the restriction $d \geq g$ is imposed in order to ensure that $\mathcal{C}^{(d)}(g-1, g-1) \neq \varnothing$. Indeed, the Hurwitz formula implies that $\mathcal{C}^{(d)}(g-1, g-1)=\varnothing$ when $d<g$, and it is not hard to see using 'staircases' that the loci $\mathcal{C}^{(d)}(g-1, g-1)$ 
are nonempty for $d \geq g$. We will prove the statement for

$$
d_{0}(g) \stackrel{\text { def }}{=} \max \{g, a(g)\}, \quad \text { where } a(g) \sim \frac{g^{3}}{54} .
$$

A convex presentation, if it exists in $\mathcal{C}^{(d)}(g-1, g-1)$, is a $(2 g+2)$-gon. Arguing as in the proof of Proposition 40, we find that it contains a strictly convex $(g+1)$-gon $P_{0}$ with vertices at lattice points, and with area strictly less than $d$. Moreover $P_{0}$ satisfies the following restriction (generalizing the adjacent edges restriction): Let $\vec{v}_{1}, \ldots, \vec{v}_{g+1}$ denote the holonomies of the edges of $P_{0}$, ordered cyclically counterclockwise and equipped with the boundary orientation of $P_{0}$. As usual we consider indices $\bmod g+1$. Then for $j=1, \ldots, 2 g+1$ and for any $1 \leq i<\frac{g+1}{2}$, the angle between $\vec{v}_{j}$ and $\vec{v}_{j+i}$ (measured counterclockwise) is less than $\pi$, and if $g+1$ is even, the angle between $\vec{v}_{j}$ and $\vec{v}_{j+(g+1) / 2}$ is $\pi$. We need to show that no such $P_{0}$ exists.

Let $a(g)$ denote the minimal area of a strictly convex $(g+1)$-gon with vertices in $\mathbb{Z}^{2}$. Then it is known (see [BT, $\left[\mathrm{Si}\right.$ ]) that $a(g) \sim \frac{g^{3}}{54}$ and $a(g) \geq g$ for $g \geq 9$. So there is nothing to prove for $g \geq 9$. If $g=3, \ldots, 8$ we need to show any strictly convex $(g+1)$-gon with vertices in $\mathbb{Z}^{2}$, of area less than $g$, does not satisfy the generalized adjacent edges restriction. This can be done by a case-by-case analysis involving Pick's formula, as in the proof of Proposition 14, we leave the details to our dedicated reader.

\section{Finding Strictly CONVEX PResentations in $\mathcal{H}(1,1)$}

7.1. Pulling back from $\mathcal{H}(2)$. One can consider $\mathcal{H}(2)$ as part of a bordification of $\mathcal{H}(1,1)$, where the boundary added corresponds to surfaces obtained by 'collapsing the two singularities.' Since having a strictly convex presentation is an open condition, one may expect that having a strictly convex presentation in $\mathcal{H}(2)$ implies having one nearby, in $\mathcal{H}(1,1)$. Indeed this turns out to be the case, as we will show in this section. For a discussion of this point of view, and a careful discussion of the inverse operation of 'splitting apart a zero', see [EMZ.

Let $P$ be a strictly convex centrally symmetric octagon with vertices $v_{0}, \ldots, v_{7}$, listed counterclockwise, and let $M$ denote the surface in $\mathcal{H}(2)$ obtained by gluing opposite sides of $P$. Denote by $e_{j}=v_{j+1}-v_{j}$ the holonomies of saddle connections representing edges of $P$ (where indices are considered mod 8). Let $u$ be a vector satisfying

$$
e_{0} \wedge u<0<e_{7} \wedge u,
$$

where $v_{1} \wedge v_{2}$ denotes the determinant of the matrix with column vectors $v_{1}, v_{2}$; in other words, $u$ lies in the convex cone generated by $e_{7}$ and $e_{0}$. For any $\varepsilon>0$, let $P_{u, \varepsilon}$ denote the centrally symmetric decagon whose vertices (listed counterclockwise) are the points

$$
v_{0}, w_{0}, v_{1}, w_{2}, v_{3}, w_{4}, v_{4}, w_{5}, v_{6}, w_{7} \text { where } w_{j} \stackrel{\text { def }}{=} v_{j}+\varepsilon u .
$$

That is we obtained $P_{u, \varepsilon}$ by splitting each of the vertices $v_{0}, v_{4}$ in two and replacing them by two nearby vertices separated by motion in the direction of $u$; and all other vertices have been moved at most a distance $\varepsilon$ from their initial positions. Since $P$ is strictly convex, the choice of direction of $u$ ensures that there exists $\delta>0$ such that for any $\varepsilon \in(0, \delta), P_{u, \varepsilon}$ is also strictly convex. See Figure 12 . 

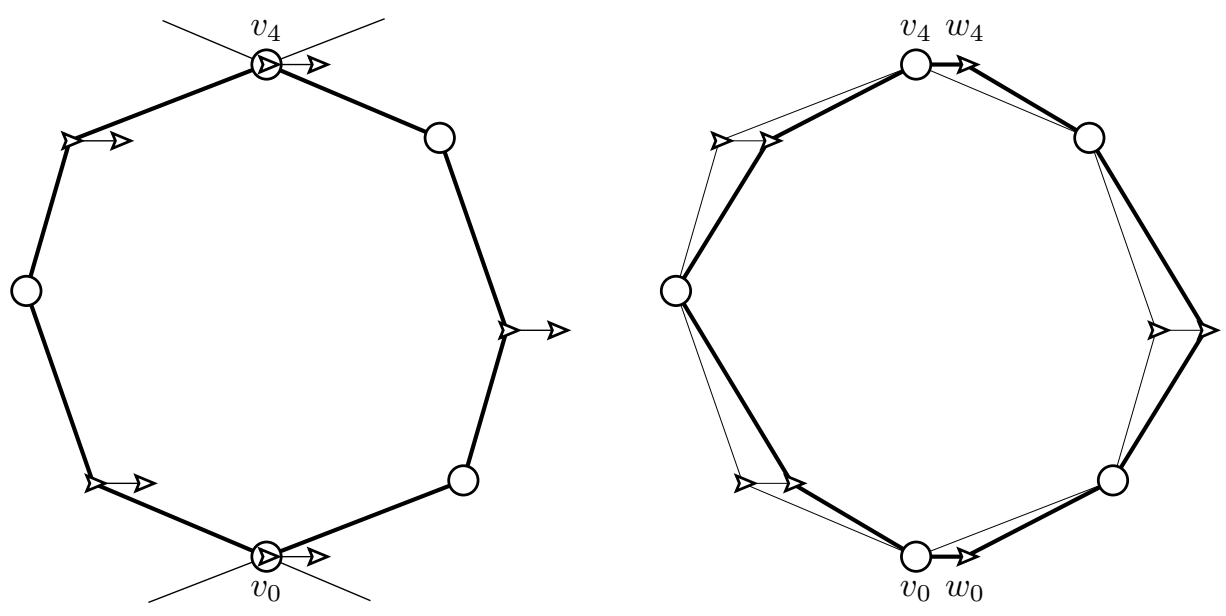

Figure 12. Octagon to decagon: $v_{0}$ and $v_{4}$ split, circle-shaped vertices $v_{j}$ stay, dart-shaped vertices $v_{j}$ move to $w_{j}$. Left: original octagon (thick) and cone of directions for $u$; right: original octagon (thin) and resulting decagon (thick). Strict convexity is preserved.

Now let $M_{u, \varepsilon}$ be the surface obtained by identifying opposite sides of $P_{u, \varepsilon}$. According to [Mc2, Cor. 5.6], the question of whether a genus two surface is an eigenform of discriminant $D$ depends only on the absolute period map $H_{1}(M, \mathbb{Z}) \rightarrow \mathbb{R}^{2}$ obtained by integrating the planar 1 -forms $d x, d y$ on $M$. Note that for any $\varepsilon>0$, the absolute period map of $M_{u, \varepsilon}$ is the same as that of $M$. Therefore $M_{u, \varepsilon} \in \mathcal{H}(1,1)$ is an eigenform of discriminant $D$ whenever $M$ is.

This implies:

Proposition 43. For any $D$, if there is a lattice surface in $\mathcal{H}(2)$ with discriminant $D, D_{0}$ or $D_{1}$, with a strictly convex presentation, then there is an eigenform surface of discriminant $D$ in $\mathcal{H}(1,1)$ with a strictly convex presentation.

\subsection{A remaining finite list of cases.}

Proposition 44. For the following values of $D$, the eigenform locus $\mathcal{E}_{D} \subset \mathcal{H}(1,1)$ contains surfaces with a strictly convex presentation:

$$
5,12,17,21,25,32,36,41,45,49,64,77,81 .
$$

Note that there are two differences between (1) and (31): discriminants 9 and 16 appear in (1) and not in (31); and all cases of the form $D_{0}, D_{1}$ are written as $D$.

Proof. For the case $D=5$ we have the regular decagon. Next we deal with the cases $17,41,49,81$. In these cases, one of the symbols $D_{0}, D_{1}$ appears in the list (1) but not the other. For example, (1) contains $41_{0}$ but not $41_{1}$. Suppose with no loss of generality that $D_{0}$ is not on the list (1), i.e. there is a lattice surface in $\mathcal{H}(2)$ corresponding to discriminant $D_{0}$, with a strictly convex presentation. Applying Proposition 43 we conclude that $\mathcal{E}_{D}$ contains a surface with a strictly convex presentation. 
For the nine remaining cases we have found strictly convex presentations by a computer search. Namely we have written a computer program, similar to the one described in $\$ 5.4$. which, given a prototype $a, b, c, e, x, y$ in $\mathcal{H}(1,1)$, checks whether the canonical decagon for this prototype is convex. This algorithm is based on the discussion of $\$ 4.2$. For each fixed $D$, there are finitely many possibilities for the discrete values $a, b, c, e$ of the prototype. For each fixed value of these, the computer program searched with a large finite number of evenly spaced values of $x$ and $y$ and stopped when a strictly convex canonical decagon was found. Parameters for strictly convex polygons obtained by this computer search are shown in Figure 13 The computer code and results of computations can be accessed ${ }^{1}$ at [LW].

\begin{tabular}{rrrrrrl}
\hline$D$ & $a$ & $b$ & $c$ & $e$ & $x$ & $y$ \\
\hline 12 & 0 & 3 & 1 & 0 & 0.9 & 0.2 \\
21 & 0 & 5 & 1 & 1 & 2.0 & 0.2 \\
25 & 0 & 3 & 2 & -1 & 0.7 & 0.2 \\
32 & 0 & 7 & 1 & 2 & 2.8 & 0.1 \\
\hline
\end{tabular}

\begin{tabular}{ccrcccc}
\hline$D$ & $a$ & $b$ & $c$ & $e$ & $x$ & $y$ \\
\hline 36 & 5 & 9 & 1 & 0 & 1.0 & 0.1 \\
45 & 0 & 9 & 1 & 3 & 3.5 & 0.1 \\
64 & 6 & 16 & 1 & 0 & 0.5 & 0.1 \\
77 & 0 & 13 & 1 & 5 & 5.8 & 0.1 \\
\hline
\end{tabular}

FiguRE 13. Parameters for strictly convex presentations in some $\mathcal{E}_{D}$ 's.

\section{Unstable convexity in $\mathcal{E}_{4,9,16}$}

We have observed that the set of surfaces admitting a strictly convex presentation is open. In this section we will prove a partial converse for the eigenform loci $\mathcal{E}_{4}, \mathcal{E}_{9}$ and $\mathcal{E}_{16}$. We will show that if $M$ is represented by a decagon $P$ which is convex but not strictly convex, then for almost every surface $M^{\prime} \in \mathcal{E}_{4,9,16}$ which is sufficiently close to $M$, the corresponding polygon $P^{\prime}$ is nonconvex. We begin by making this precise.

Recall that for any $D$, the eigenform locus $\mathcal{E}_{D}$ is a closed suborbifold of $\mathcal{H}(1,1)$, of real dimension 5. A marked decagon is a decagon (not necessarily convex) equipped with a partition of its edges into pairs, such that paired edges are parallel and of equal length. There is an obvious topology on the space of marked decagons. Identifying paired edges maps each marked decagon $P$ to a translation surface $M_{P}$, and this map $P \mapsto M_{P}$ is a homeomorphism between a neighborhood of $M$ in $\mathcal{H}(1,1)$ and a neighborhood of $P$ in the space of marked decagons. We will denote this neighborhood by $\mathcal{U}=\mathcal{U}(M, P)$, and denote the inverse map by $M \mapsto P_{M}$. Thus, if $M=M_{P} \in \mathcal{U}$ and $M^{\prime} \in \mathcal{U}$ is close to $M$, then $P^{\prime}=P_{M^{\prime}}$ is close to $P$ and $M^{\prime}=M_{P^{\prime}}$.

Proposition 45. Suppose $D=4,9$ or 16 . Suppose $P$ is a convex marked decagon such that $M_{P} \in \mathcal{E}_{D}$ and $\mathcal{U}=\mathcal{U}(M, P)$ is as above. Then there is a neighborhood $\mathcal{U}_{0} \subset \mathcal{U}$ of $M$ such that

$$
\left\{M^{\prime} \in \mathcal{U}_{0} \cap \mathcal{E}_{D}: P_{M^{\prime}} \text { is convex }\right\}
$$

is a four-dimensional submanifold.

\footnotetext{
${ }^{1}$ Do not see the references therein! Due to risk of infinite regress, the reader is advised to proceed with care.
} 
We preface the proof of this proposition with some remarks about the structure of strata and the structure of the loci $\mathcal{E}_{D}$. More details can be found in [Z] and [Mc2]. Let $S$ be a model compact orientable surface of genus two with a set $\Sigma=\left\{\sigma_{1}, \sigma_{2}\right\}$ consisting of two distinguished points of $S$. The stratum $\mathcal{H}(1,1)$ is locally modelled on the cohomology group $H^{1}\left(S, \Sigma ; \mathbb{R}^{2}\right)$, and the eigenform locus $\mathcal{E}_{D}$ is modelled on the semi-direct product group $G \ltimes \mathbb{R}^{2}$ (where $\mathbb{R}^{2}$ represents motion in the rel leaves). More precisely, for any $M \in \mathcal{H}(1,1)$ there is a neighborhood $\mathcal{U}$ of $M$ in $\mathcal{H}(1,1)$, and a homeomorphism of $M$ and $S$ mapping the singularities of $M$ to $\Sigma$, such that each $M^{\prime} \in \mathcal{U}$ is uniquely determined by the holonomies $\operatorname{hol}\left(\alpha, M^{\prime}\right)$ obtained by integrating the translation structure along $\alpha$, for any curve $\alpha$ in $S$ representing a relative one-cycle in $H_{1}(S, \Sigma)$. Moreover (making $\mathcal{U}$ smaller if necessary), there is a small neighborhood $\mathcal{V}$ of the identity in $G \ltimes \mathbb{R}^{2}$, such that for any $M^{\prime} \in \mathcal{E}_{D} \cap \mathcal{U}$ there are unique $(g, v) \in \mathcal{V}$, such that for any 1-cycle $\alpha$,

$$
\operatorname{hol}\left(\alpha, M^{\prime}\right)=\left\{\begin{aligned}
g \cdot \operatorname{hol}(\alpha, M) & \text { if } \alpha \text { is a closed curve in } S \\
g .(\operatorname{hol}(\alpha, M)+v) & \text { if } \alpha \text { goes from } \sigma_{1} \text { to } \sigma_{2}
\end{aligned}\right.
$$

Here $g \cdot \vec{u}$ denotes the image of $\vec{u} \in \mathbb{R}^{2}$ under the linear map $g$. We will denote the surface corresponding to $(g, v)$ as above by $(g, v) . M$.

The idea of proof will be to show that given any $P$, there is a one-dimensional linear subspace $L \subset \mathbb{R}^{2}$, such that if $(g, v) \in \mathcal{V}$ is as above, and $v \notin L$, then the decagon $P_{M^{\prime}}$ corresponding to $M^{\prime}=(g, v) . M$ is not convex. That is, all motions in the rel leaf except possibly motions in a particular direction destroy convexity. We proceed to the details.

Proof. We take $\mathcal{U}_{0}$ small enough so that it is contained in the neighborhood as in the preceding paragraph. That is, the notation $(g, v) . M$ makes sense in $\mathcal{U}_{0}$, for $(g, v) \in \mathcal{V}$, and so does the notation $P_{M^{\prime}}$. It follows from the preceding discussion that $P_{(g, v) . M}$ is obtained from $P_{M}$ as follows. We first subdivide the vertices of $P$ into two classes, according to whether they map to the singularity labelled $\sigma_{1}$ or $\sigma_{2}$ in $M$. We then keep the vertices mapping to $\sigma_{1}$ fixed and move all of the others by adding $v$. Connecting the vertices, this defines a new polygon $P(v)$ if $v$ is sufficiently small (and we can assume this by making $\mathcal{U}_{0}$ smaller). We then apply the map $g$ to $P(v)$. The resulting polygon is $P_{(g, v) . M}$.

Since linear maps in $G$ preserve convexity, in order to prove the proposition it suffices to show that for any convex marked decagon $P$, for which $M_{P} \in \mathcal{E}_{4,9,16}$, the set of $v$ for which $P(v)$ is convex, are contained in a one-dimensional linear subspace. To show this, it suffices to show that for any $P$, there are three or more edges of $P$ which are consecutive and parallel, and connect distinct singularities; for then any rel perturbation $v$ which is not in the direction of these edges will result in $P(v)$ being non-convex. E.g. in the last polygon in Figure 14 (a square), this occurs in the top and bottom of the figure. If we move the black points up (respectively down) with respect to the white points, convexity will be destroyed at the vertex between edges $b$ and $c$ (respectively between $a$ and $b$ ) in the top of the figure, so any rel operation which does not move points horizontally will destroy convexity.

The proof of existence of three parallel consecutive edges proceeds by a caseby-case analysis. We will show that the only marked decagons which represent surfaces in $\mathcal{H}(1,1)$ are those shown in Figures 14 and 15 . Since, by $\$ 6$, surfaces in $\mathcal{E}_{4,9,16}$ have no strictly convex presentations, the decagon which is the first polygon 

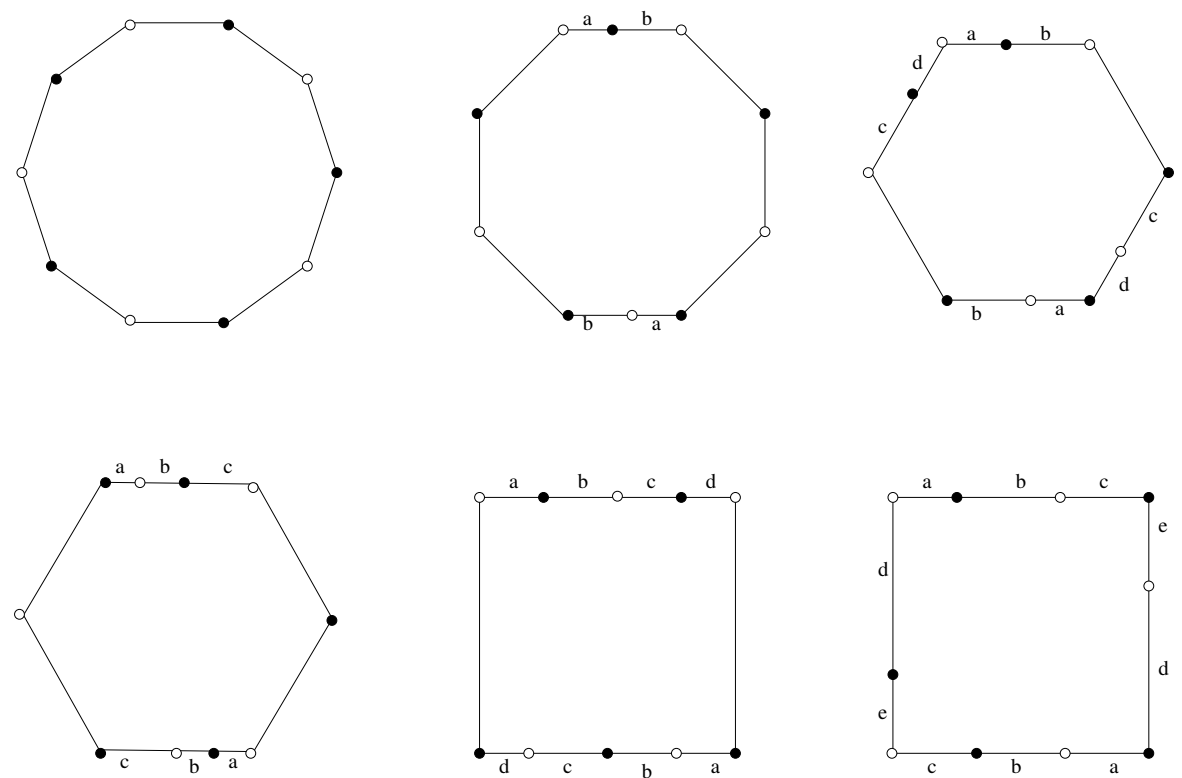

FiguRE 14. The standard marked decagon, with several convex realizations in $\mathcal{H}(1,1)$. The top row cannot appear in $\mathcal{E}_{4,9,16}$.

in Figure 14 does not appear in these eigenform loci. Similarly, the octagon and hexagon in the top row of Figure 14 also do not appear in $\mathcal{E}_{4,9,16}$, since they can be made into a strictly convex decagon by a rel perturbation, which would again contradict $\$ 6$. All the other polygons in the figure satisfy our claim. This will conclude the proof.
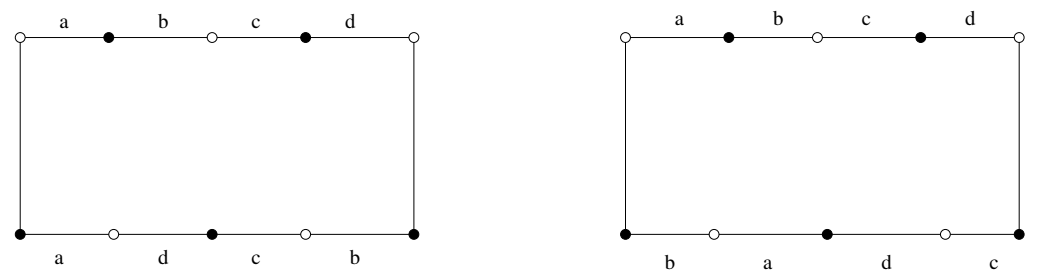

FiguRE 15. Two additional marked decagons, realized as cylinders in $\mathcal{H}(1,1)$.

When realized as a convex polygon in the plane, some sides of the marked decagon become parallel. This gives a partition of the set of edges into subsets of parallel edges, where each subset is composed of some pairs of edges identified in the marked decagon (but possibly more than one pair). Considering the different possibilities for these partitions, one finds the following possibilities:

(a) a strictly convex decagon.

(b) a strictly convex octagon in which a pair of parallel edges are each a concatenation of two consecutive edges of $P$.

(c) a strictly convex hexagon in which two pairs of parallel edges are each a concatenation of two consecutive edges of $P$. 
(d) a strictly convex hexagon in which a pair of parallel edges are each a concatenation of three consecutive edges of $P$.

(e) a parallelogram in which a pair of parallel edges is each a concatenation of four consecutive edges of $P$.

(f) a parallelogram in which one pair of parallel edges is each a concatenation of three consecutive edges of $P$, and one pair of parallel edges is each a concatenation of two consecutive edges of $P$.

We will go over these cases one by one and show that all markings which give rise to a surface in $\mathcal{H}(1,1)$ appear in Figures 14 and 15 . Note that the total cone angle around singularities for a surface in $\mathcal{H}(1,1)$ is $8 \pi$ and hence all vertices of the decagon must be singularities of the translation surface. All indices in the discussion below will be given in cyclic order along the boundary of $P$. Also note that if $a_{1}, a_{2}$ are consecutive and $b_{1}, b_{2}$ are consecutive and parallel then we cannot glue $a_{1}$ to $b_{2}$ and $a_{2}$ to $b_{1}$ because then the vertex between $a_{1}$ and $a_{2}$ will have cone angle $2 \pi$, i.e. will not be a singularity, and we will not be in $\mathcal{H}(1,1)$. We will call this the basic restriction.

Clearly case (a) corresponds to the first polygon in Figure 14 In case (b), suppose $a_{1}, a_{2}$ and $b_{1}, b_{2}$ are two pairs of consecutive edges whose concatenations are parallel edges of the octagon. If $a_{1}$ is glued to $b_{1}$ and $a_{2}$ to $b_{2}$ in $P$ then we have the second polygon in Figure 14. The other case is forbidden by the basic restriction. By the same reasoning, in case (c), we can only glue sides as in the third polygon in Figure 14 .

In case (d), let the three pairs of consecutive edges be labelled $a_{1}, a_{2}, a_{3}$ and $b_{1}, b_{2}, b_{3}$. The gluing in Figure 14 corresponds to identifying $a_{i}$ with $b_{i}$. If $a_{1}$ is identified with $b_{3}$ then by the basic restriction we must identify $a_{2}$ with $b_{2}$ and $a_{3}$ with $b_{1}$. Now consider the vertex on $a_{3}$ which is not adjacent to $a_{2}$. Following the identifications we see that it is identified with three other vertices and has a total angle of $\pi$, so we are not in $\mathcal{H}(1,1)$. By similar reasoning $a_{3}$ cannot be identified with $b_{1}$. Since $a_{1}$ and $a_{3}$ cannot both be identified with $b_{2}$, we have that for $i=1$ or $i=3, a_{i}$ is identified with $b_{i}$. But then the basic restriction forces the gluing to be as in Figure 14

In case (e), all three cases of Figures 14 (fifth polygon) and 15 are possible. To see that there are no others, let $a_{1}, \ldots, a_{4}, b_{1}, \ldots, b_{4}$ be the labelling of the four consecutive edges. If $a_{1}$ is identified with $b_{4}$ and $a_{4}$ with $b_{1}$ then the point vertex on $a_{1}$ on the side opposite to $a_{2}$ becomes a regular point and we are not in $\mathcal{H}(1,1)$. If $a_{1}$ is identified with $b_{4}$ and $a_{4}$ with $b_{2}$ then by the basic restriction we must identify $a_{3}$ with $b_{3}$ and hence $a_{2}$ with $b_{1}$, which violates the basic restriction for the pair $a_{3}, a_{4}$. If $a_{1}$ is identified with $b_{4}$ and $a_{4}$ with $b_{3}$ then by the basic restriction for the sides $a_{2}, a_{3}$ we have the polygon on the left side of Figure 15 . So we have dealt with all cases in which $a_{1}$ is identified with $b_{4}$, and by symmetry, when $a_{4}$ is identified with $b_{1}$. Now suppose $a_{1}$ is identified with $b_{2}$ and $a_{4}$ with $b_{3}$. By the basic restriction $a_{2}$ is identified with $b_{4}$ and $a_{3}$ with $b_{1}$. Then the vertex between $b_{2}$ and $b_{3}$ becomes a regular point and we are not in $\mathcal{H}(1,1)$. The remaining case is $a_{1}$ identified with $b_{3}$ and $a_{4}$ with $b_{2}$. If $a_{2}$ is identified with $b_{4}$ we have the polygon on the right side of Figure 15 and if $a_{2}$ is identified with $b_{1}$ then the vertex between $a_{2}$ and $a_{3}$ becomes a regular point. 
In case (f), applying the basic restriction to the pair of consecutive sides $a_{1}, a_{2}$, $b_{1}, b_{2}$ which form a side of the hexagon, we see that we must identify $a_{i}$ with $b_{i}$. Then the discussion reduces to case (d).

Corollary 46. Let $D=4,9$ or 16 . With respect to the measure of Theorem 20, almost every surface in $\mathcal{E}_{D}$ has no convex presentation.

Proof. In order to prove that a set of translation surfaces has measure zero, it is enough to show that it has measure zero in the cover of $\mathcal{H}(1,1)$ corresponding to translation surfaces equipped with a marking, i.e. a fixed identification of $M$ with $S$ such that singularities map to $\Sigma$. Fixing a marking, we note that the possible edges of convex polygons $P$ may be labelled by homotopy classes of curves beginning and ending at $\Sigma$. In particular there are countably many ways of using a marked decagon $P$ to get a convex presentation. For each such marked decagon $P$ and each surface $M$ constructed via $P$ with a convex presentation, we have exhibited a neighborhood of $M$ in which the set of surfaces for which $P$ is convex, is of measure zero. The result follows using the fact that a countable union of sets of measure zero has measure zero.

\section{Proof of theorem 2}

Assertions (i) and (ii) were proved in $\$ 6$ and $\$ 7$ respectively. To deduce (iii) from (ii), note that if $D$ is not a square, and is not equal to 5 , by Theorem 21. any $G$-orbit in $\mathcal{E}_{D}$ is dense in $\mathcal{E}_{D}$. Moreover in case $D=5$ the only orbit which is not dense in $\mathcal{E}_{5}$ is that of the regular decagon, which is clearly strictly convex. Thus the $G$-orbit of any surface which is neither arithmetic, nor contained in $\mathcal{E}_{4,9,16}$, contains a surface with a strictly convex presentation. This concludes the proof in light of $G$-invariance. Assertion (iv) follows from Corollary 27. To prove (v) note that the collections of lattice surfaces in $\mathcal{E}_{4,9,16}$ consists of a countable collection of $G$-orbits, so in particular is of measure zero. In view of Corollary 46, almost every surface in $\mathcal{E}_{4,9,16}$ has the required properties.

\section{Another Question of Veech}

Theorem 2(v) resolves a question of Veech [V2, Question 5.2]. Namely Veech asked whether there are non-lattice surfaces with no convex presentations, and as we have seen, almost every surface in $\mathcal{E}_{4,9,16}$ has these properties. In case of an affirmative answer, Veech asked a more refined question. Namely (in our notations), given $g$, let $\mathcal{H}$ denote the hyperelliptic component of one of the strata $\mathcal{H}(g-1, g-$ $1), \mathcal{H}(2 g-2)$, let $\mathcal{H}_{\mathrm{nc}}$ denote the subset of surfaces without convex presentations, and let $\overline{\mathcal{H}_{\mathrm{nc}}}$ denote the closure of $\mathcal{H}_{\mathrm{nc}}$. Is there a surface in $\mathcal{H}_{\mathrm{nc}}$ whose orbit-closure is $\overline{\mathcal{H}_{\text {nc }}}$ ? Our analysis shows that in the stratum $\mathcal{H}(1,1)$, the answer to this question is negative. Namely, by Theorem 2 (iii, iv) $\mathcal{H}_{\text {nc }}$ consists of:

(a) Almost every surface in $\mathcal{E}_{4,9,16}$.

(b) A finite list of arithmetic surfaces.

Therefore $\overline{\mathcal{H}_{\mathrm{nc}}}$ consists of $\mathcal{E}_{4,9,16}$, and a finite list of closed $G$-orbits. Since $\mathcal{E}_{4}, \mathcal{E}_{9}$ and $\mathcal{E}_{16}$ are closed and disjoint, part (a) of this list shows a negative answer to Veech's question in $\mathcal{H}(1,1)$. We remark that the list in (b) is nonempty. In light of Proposition 14, we know that there are at least three $G$-orbits of arithmetic surfaces outside $\mathcal{E}_{4,9,16}$ without strictly convex presentations. Namely, this will be satisfied by the $G$-orbit of any square tiled surface made of $d \leq 7$ squares, which is not in 
$\mathcal{E}_{4,9,16}$. Since 5 and 7 are prime we can use $d=5,7$ to get two examples in $\mathcal{E}_{49}$ and one in $\mathcal{E}_{25}$.

\section{REFERENCES}

[B] M. Bainbridge, Billiards in L-shaped tables with barriers, Geom. Func. Anal. 20 (2010) 299-356.

[BT] I. Bárány and N. Tokushige, The minimum area of convex lattice $n$-gons, Combinatorica 24(2) (2004) 171-185.

[BM] I. Bouw and M. Möller, Teichmüller curves, triangle groups, and Lyapunov exponents Ann. of Math. (2) 172 (2010), no. 1, 139-185.

[C] K. Calta, Veech surfaces and complete periodicity in genus 2, J. Amer. Math. Soc. 17 (2004) 871-908.

[CS] K. Calta and J. Smillie, Algebraically periodic translation surfaces, J. Mod. Dyn. 2 (2008) $209-248$

[EMZ] A. Eskin, H. Masur and A. Zorich, Moduli spaces of abelian differentials: the principal boundary, counting problems, and the Siegel-Veech constants Publ. I. H. E. S. 97 (2003) 631-678.

$[\mathrm{EM}]$ A. Eskin and M. Mirzakhani, Invariant and stationary measures for the $\mathrm{SL}(2, \mathbb{R})$-action on moduli space preprint (2012), available at http://www.math.uchicago.edu/ ${ }^{\text {eskin/ }}$

[EMM] A. Eskin, M. Mirzakhani and A. Mohammadi, Isolation, Equidistribution and Orbit Closures for the $\mathrm{SL}(2, \mathbb{R})$-action on moduli space, preprint (2012), available online at http://www.math.uchicago.edu/ ${ }^{\sim}$ eskin/

$[\mathrm{H}]$ W. P. Hooper, Grid graphs and lattice surfaces, preprint (2009), available at http://wphooper.com/docs/papers/

[HLM] P. Hubert, E. Lanneau and M. Möller, Completely periodic directions and orbit closures of many pseudo-Anosov Teichmüller discs in $\mathcal{Q}(1,1,1,1)$, Math. Ann. 353 (2012), no. 1, $1-35$.

[HL] P. Hubert and S. Lelièvre, Prime arithmetic Teichmller discs in $\mathcal{H}(2)$, Israel J. Math. 151 (2006), 281-321.

[HS] P. Hubert and T. Schmidt, An introduction to Veech surfaces, in Handbook of Dynamical Systems, Vol 1B B. Hasselblatt and A. Katok (eds.) Elsevier (2006) p. 501-526.

[KZ] M. Kontsevich and A. Zorich, Connected components of the moduli spaces of Abelian differentials with prescribed singularities, Invent. Math. 153 (2003), no. 3, 631-678.

[LW] S. Lelièvre and B. Weiss, Translation surfaces with no convex presentations, Files attached to source, http://arxiv.org

[MT] H. Masur and S. Tabachnikov, Rational billiards and flat structures, in Handbook of dynamical systems, Enc. Math. Sci. Ser. (2001).

[Ma] C. Matheus, Disquisitiones mathematicae, http://matheuscmss.wordpress.org

[Mc1] C. T. McMullen, Billiards and Teichmüller curves on Hilbert modular surfaces J. Amer. Math. Soc. 16 (2003) 857-885.

[Mc2] C. T. McMullen, Dynamics of $\mathrm{SL}_{2}(\mathbb{R})$ over moduli space in genus two, Ann. Math. (2) 165 (2007), no. 2, 397-456.

[Mc3] C. T. McMullen, Teichmüller curves in genus two: Discriminant and spin, Math. Ann. 333:1 (2005), 87-130.

[Mo] T. Monteil, A homological condition for a dynamical and illuminatory classification of torus branched coverings, (2006) http://arxiv.org/pdf/math/0603352v1.pdf

[P] Pick's theorem, http://en.wikipedia.org/wiki/Pick's_theorem

[S] G. Schmithüsen, An algorithm for finding the Veech group of an origami, Mathematics 13 No.4 (2004) 459-472.

[Si] R. J. Simpson, Convex lattice polygons of minimum area, Bull. Austral. Math. Soc., 42(3) (1990) 353-367.

[V1] W. A. Veech, Teichmüller curves in moduli space, Eisenstein series and an application to triangular billiards, Invent. Math. 97 (1989), no. 3, 553-583.

[V2] W. A. Veech, Geometric realizations of hyperelliptic curves, in Algorithms, fractals, and dynamics (Okayama/Kyoto, 1992), Y. Takahashi (ed.), p. 217-226, Plenum, (1995). 
[Vi] M. Viana, Ergodic theory of interval exchange maps, Revista. Mat. Comp. 19 (2006) $7-100$.

[W] C. C. Ward, Calculation of Fuchsian groups associated to billiards in a rational triangle, Erg. Th. Dynam. Sys. 18 (1998), no. 4, 1019-1042.

[Wr] A. Wright, Schwartz triangle mappings and Teichmüller curves: the Veech-Ward-BouwMöller curves, preprint (2012), to appear in Geom. Funct. Ana.

[Zo] A. Zorich, Flat surfaces, in Frontiers in number theory, physics and geometry, P. Cartier, B. Julia, P. Moussa and P. Vanhove (eds), Springer (2006).

Laboratoire de mathématique d'Orsay, Umr 8628 Cnrs/Université Paris-Sud, Bât. 425, CAmpus Orsay, 91405 Orsay CEdex, France, samuel.lelievre@math.u-psud.fr

Ben Gurion University, Be'er Sheva, Israel 84105 barakw@math.bgu.ac.il 